\title{
L'érosion différentielle dans les reliefs de l'Estrie et de la Nouvelle-Angleterre, entre Montréal et le piedmont sud-est des White Mountains
}

\section{The Influence of Differential Erosion on the Relief of the} Eastern Townships and of New England between Montréal and the Southeast Piedmont of the White Mountains Der Einfluss von Differentialerosion auf das Relief der Estrie
und das der Nouvelle-Angleterre zwischen Montréal und dem
Östlichen Vorgebirge der White Mountains

Pierre Birot, Alain Godard et Jean Pelletier

Volume 37, numéro 1, 1983

URI : https://id.erudit.org/iderudit/032495ar

DOI : https://doi.org/10.7202/032495ar

\section{Aller au sommaire du numéro}

\section{Éditeur(s)}

Les Presses de l'Université de Montréal

\section{ISSN}

0705-7199 (imprimé)

1492-143X (numérique)

\section{Découvrir la revue}

\section{Citer cet article}

Birot, P., Godard, A. \& Pelletier, J. (1983). L'érosion différentielle dans les reliefs de l'Estrie et de la Nouvelle-Angleterre, entre Montréal et le piedmont sud-est des White Mountains. Géographie physique et Quaternaire, 37(1), 3-25. https://doi.org/10.7202/032495ar

\section{Résumé de l'article}

Entre Montréal et Granby, dans une véritable plaine à inselbergs, ces derniers correspondent à des intrusions tantôt syénitiques, tantôt basiques : ils sont plus résistants que les calcaro-schistes cambro-ordoviciens. L'intrusion la plus orientale est celle du mont Mégantic, qui comporte un croissant syénitique enveloppant une bosse de granite. Les intrusions de granite dévonien de l'Estrie et du nord du Vermont sont souvent en creux, ce qui peut s'expliquer soit par leur composition granodioritique (alvéole d'Island Pond), soit par une intense microfissuration (alvéole de Scotstown). La position culminante des White Mountains paraît due (en grande partie) à l'érosion différentielle. Les séries de Littleton, où les unités schisteuses sont aussi résistantes que les éléments quartzitiques et gneissiques. dominent, au nord, les gneiss olivériens, dont la position déprimée est attribuable à leur fracturation, non suivie de recristallisation, et ailleurs, les granites de Bickford, pourtant relativement acides. En position culminante se placent également les syenites et les granites à hastingsite mésozoïques. Quant aux granites dits de Conway, tantôt ils sont troués par d'amples cuvettes, tantôt ils constituent des fiers monolithes limités par des diaclases espacées. C'est que ces roches sont très hétérogènes, tantôt blanches et acides, tantôt grises et riches en biotite et en hornblende, ainsi qu'en plagioclases. Au total, les roches occupant le sommet de l'échelle de dureté sont celles où les perthites et les microperthites constituent une trame continue. Pour les autres variétés de roches, le degré de microfissuration et la composition minéralogique jouent alternativement le rôle principal. 


\section{L'ÉROSION DIFFÉRENTIELLE DANS LES RELIEFS DE L'ESTRIE ET DE LA NOUVELLE-ANGLETERRE, ENTRE MONTRÉAL ET LE PIEDMONT SUD-EST DES WHITE MIOUNTAINS}

Pierre BIROT, Alain GODARD et Jean PELLETIER, les deux premiers auteurs, laboratoire de Géographie physique (L. A. 141, C.N.R.S.), 191, rue Saint-Jacques, 75005 Paris, France, et le troisième auteur, laboratoire de Géographie rhodanienne (L.A. 260, C.N.R.S.), avenue de l'Université, 69500 Bron, France.

RÉSUMÉ Entre Montréal et Granby, dans une véritable plaine à inselbergs, ces derniers correspondent à des intrusions tantôt syénitiques, tantôt basiques: ils sont plus résistants que les calcaroschistes cambro-ordoviciens. L'intrusion la plus orientale est celle du mont Mégantic, qui comporte un croissant syénitique enveloppant une bosse de granite. Les intrusions de granite dévonien de l'Estrie et du nord du Vermont sont souvent en creux, ce qui peut s'expliquer soit par leur composition granodioritique (alvéole d'Island Pond), soit par une intense microfissuration (alvéole de Scotstown). La position culminante des White Mountains paraît due (en grande partie) à l'érosion diffẻrentielle. Les séries de Littleton, où les unités schisteuses sont aussi résistantes que les éléments quartzitiques et gneissiques, dominent, au nord, les gneiss olivériens, dont la position déprimée est attribuable à leur fracturation, non suivie de recristallisation, et ailleurs, les granites de Bickford, pourtant relativement acides. En position culminante se placent également les syénites et les granites à hastingsite mésozoïques. Quant aux granites dits de Conway, tantôt ils sont troués par d'amples cuvettes, tantôt ils constituent des fiers monolithes limités par des diaclases espacées. C'est que ces roches sont très hétérogènes, tantôt blanches et acides, tantôt grises et riches en biotite et en hornblende, ainsi qu'en plagioclases. Au total, les roches occupant le sommet de l'échelle de dureté sont celles où les perthites et les microperthites constituent une trame continue. Pour les autres variétés de roches, le degré de microfissuration et la composition minéralogique jouent alternativement le rôle principal.
ABSTRACT The influence of differential erosion on the relief of the Eastern Townships and of New England between Montreal and the southeast piedmont of the White Mountains. Between Montréal and Granby, the studied profile cuts across a true erosion plain where inselbergs coincide with syenitic and basic intrusions more resistant than the Cambro-Ordovician calcareous shales and schists. Mount Mégantic, the most eastern intrusion consists of a syenitic crescent and a granitic boss. In the Eastern Townships and northern Vermont, Devonian granitic intrusions are often hollowed out into basins. This derives either from their granodioritic composition (Island Pond) or from severe microfissuration (Scotstown). The prominent position of the White Mountains seems to be due to selective erosion. The Littleton Series, where schistous units are as resistant as the quartzitic and gneissic elements, rise on their northern boundary above the oliverian gneisses. These were easily eroded since no recrystallization occurred after fracturing. Elsewhere, the Littleton Series rise above Bickford granites despite the rather acid composition of the latter. Mesozoic hastingsite syenites and granites also have a prominent position. As for the granites classified as Conway granites, they range from white and acid varieties to grey varieties with a high content of plagioclases, biotites and hornblende. This heterogeneity may explain the topographic contrast between huge basins and monoliths, the latter being limited by spaced joints. The rocks at the top of the hardness scale are those where the main components, perthites and microperthites, form the structural grain. As for the other types of rocks, the degree of microfissuration (determined after microscopic examination and measurement of the filtration rate of

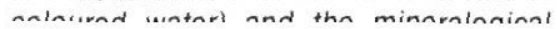

ZUSAMMENFASSUNG Der Einfluss von Differentialerosion auf das Relief der Estrie und das der Nouvelle-Angleterre zwischen Montréal und dem Östlichen Vorgebirge der White Mountains. Zwischen Montréal und Granby überquert das erforschte Profil eine echte Erosionsebene, wo Inselberge mit syenetischen und basischen Intrusionen zusammenfallen, die widerstandsfähiger als die Cambro -Ordovician Kalkär Schalen und Schiefer sind. In der Estrie und im nördlichen Vermont, sind devoniasniche Graniti intrusionen oft zu Becken ausgehölt. Das kommt entweder von ihrer granodioritischen Zusammensetzung (Island Pond) oder von häftiger Mikrospaltung (Scotstown) her. Die hervorstehende Stellung der White Mountains scheint hauptsächlich, aber nicht ausschliesslich, von selektiver Erosion zu stammen. Die Littleton Series, wo schieferhaltige Einheiten aus noch unbekannten Gründen, ebenso widerstandsfähig wie die Quartz- und Gneisselemente sind, erheben sich an ihrer nördlichen Grenze über die oliveranischen Gneisse. Die wurden leicht erodiert weil es nach der Frakturation keine Rekristalisation gab. Anderswo erheben sich die Littleton Series über Bickford Granite, trotz deren ziemlich saurer Beschaffenheit. Diese Mannigfaltigkeit kann vielleicht den topographischen Kontrast zwischen grossen Becken und Monolithen erklären, letztere findet man hauptsächlich in durch Fugen begrenzten Randstellungen. Das Gestein am Gipfel der Härteskala, hat als Hauptkomponenten Bethite und Mikroperthite, die, die strukturelle Körnung bilden. Für die anderen Gesteinstypen kommen der Grad der Mikrospaltung (bestimmt durch mikroskopische Prüfung und Messung der Filtrationsgeschwindigkeit von gefärbtem Wasser) und die mineralogische Zusammensetzung abwechselnd ale Hairntalamant irnr 
La Nouvelle-Angleterre et l'Estrie comportent de nombreux reliefs parfois de grande vigueur (Mount Washington, mont Mégantic, Percy Peaks) développés dans les roches cristallines extrêmement variées par leur origine et leur composition minéralogique, qui constituent cette partie des Appalaches.

Dans le but de mieux comprendre les relations entre relief et érosion différentielle, l'itinéraire choisi comporte une double traversée du bourrelet appalachien depuis les basses terres du Saint-Laurent, surmontées par les collines Montérégiennes, jusqu'au piedmont de la Nouvelle-Angleterre, en contrebas des Ossipee Mountains.

Cette étude n'a qu'un caractère exploratoire, tant en raison du caractère souvent linéaire du parcours que du nombre relativement faible des échantillons recueillis (150 environ).

Ces derniers ont fait l'objet, après détermination des caractères pétrographiques classiques, d'une appréciation de leur perméabilité ; cette dernière reposant sur les tests suivants: 1) L'examen microscopique de la fissuration pour toutes les plaques minces. 2) De nombreux essais de pénétration par le soluchrome, tant sur le terrain qu'en laboratoire. II n'a été tenu compte que des échantillons superficiels pour lesquels la pénétration était faible ou nulle ou encore des échantillons de carrière. II est bien évident que les échantillons superficiels peuvent porter sur un volume exceptionnellement peu perméable et que certains échantillons de carrière ont pu être perturbés par l'exploitation. 3) Par ailleurs, nous disposons d'un très petit nombre de porométries au mercure et de mesures de perméabilité à l'eau par la méthode Berneix (effectuées au laboratoire de Géographie physique - L.A. 141 CNRS - de Paris).

La région ayant été recouverte à plusieurs reprises par l'inlandsis ou les glaciers régionaux, il a fallu modifier quelque peu l'emploi des méthodes habituelles. L'action glaciaire entraîne en effet de sérieux inconvénients :

- Tout d'abord dans la plupart des cas, les altérites ont été partiellement déblayées, ce qui interdit toute observation du passage graduel de la roche saine à l'altérite in situ.

- Fait plus grave, les produits de ce balayage étalés sous forme d'un placage de drift masquent le fond de nombreuses cuvettes, en particulier sur le piedmont sud des White Mountains.

- Notons aussi que les escarpements de taille moyenne, visibles sur les flancs de certaines vallées peuvent résulter de coups de gouge latéraux donnés par la glace en mouvement, sans correspondre nécessairement à des roches très résistantes.

- Toutefois, l'évacuation des altérites n'a pas été totale: en plusieurs endroits subsistent des volumes d'arènes épargnés par la glace et qui peuvent mesurer plusieurs mètres d'épaisseur. C'est le cas notamment au mont Mégantic dans les ensembles basiques de la dépression semi-annulaire. Aux environs de Conway, une masse d'arènes granitiques épaisse parfois de 4 à 5 $\mathrm{m}$ est fossilisée sous la moraine.

Ajoutons enfin que le nettoyage glaciaire ne présente pas que des inconvénients: il peut en certains cas faciliter l'échantillonnage des roches saines dans les dépressions (dans la cuvette de la Nulhegan River par exemple).

\section{I - PRINCIPALES UNITÉS RÉGIONALES}

\section{A. LA PLAINE À INSELBERGS DE LA RÉGION ENTRE MONTRÉAL. ET GRANBY}

C'est un paysage de physionomie tropicale que nous retrouvons à la latitude de $45^{\circ}$ et sous un climat aux hivers rudes et neigeux.

Le chapelet des intrusions dites montérégiennes d'âge crétacé domine de 200 à $400 \mathrm{~m}$ une plaine qui recoupe une structure rubannée orientée NNE-SSW, correspondant à l'affleurement d'abord de simples ondulations, puis de plis et d'écailles affectant les couches cambro-ordoviciennes (coupe 1, fig. 1).

Le secteur le plus plan coïncide approximativement avec les argilites et les calcaires impurs ordoviciens. Mais l'impression de planitude est due en partie au comblement de dépressions préglaciaires et glaciaires par des dépôts morainiques, lacustres, et surtout marins (mer de Champlain).

En direction du S-SE, on passe à un relief de croupes parallèles développées dans des schistes plus métamorphiques, avec quelques intercalations de quartzites. Les plus résistants affleurent dans l'axe anticlinal des Green Mountains, qui appartenaient déjà à la zone de la biotite. Le même degré de métamorphisme affecte les séries siluriennes et dévoniennes plus orientales, qui comportent quelques intercalations de quartzites. Tout cet ensemble est basculé en direction du NE, où les différences lithologiques s'expriment de plus en plus faiblement.

La mise en creux des calcaires par rapport à toutes les autres catégories de roches, même schisteuses, est un fait général dans l'ensemble des Appalaches; il suggère la prédominance de l'érosion chimique au cours d'une période de stabilité tectonique très prolongée qui aurait permis le développement de la surface d'aplanissement de Montréal, au moins dans la partie la plus basse d'un bloc basculé vers l'ouest. Nous ne savons pas si ce dernier était lui-même tronqué par une surface d'aplanissement régulière plus ancienne.

Le schéma proposé par R. BLANCHARD (1960) suivant lequel la surface d'aplanissement de Montréal, serait emboîtée dans une surface d'aplanissement plus ancienne située au SE, n'est pas vérifié ici puisqu'on s'élève insensiblement dans cette dernière direction. Les remaniements dus d'une part à l'érosion glaciaire et d'autre part à l'accumulation fluvio-glaciaire ne permettent pas une analyse cyclique aussi fine, puisque les dénivellations, dans les deux cas, peuvent atteindre quelques dizaines de mètres. 
Les intrusions circulaires (PERRAULT, 1970) donnent des inselbergs de dureté typiques, évoquant étrangement les paysages de la zone chaude. Ils dominent une surface d'aplanissement parfaitement régulière jusqu'au méridien de Waterloo. II est vrai que la roche encaissante est formée de schisto-calcaires peu résistants.

Les points les plus hauts se situent généralement à la périphérie, même lorsque des roches variées affleurent dans l'anneau externe.

L'inselberg de Saint-Hilaire (dénivellations supérieures à $300 \mathrm{~m}$ ) présente une dissymétrie inverse de celle que permettrait de prévoir la composition minéralogique. En effet, le versant $\mathrm{W}$ et $\mathrm{SW}$ le plus raide est composé d'essexite où les feldspaths forment de 50 à $70 \%$ de la roche, se partageant à peu près également entre des plagioclases (An 15-40) et les alcalins. Les éléments ferromagnésiens sont représentés, en parts à peu près égales, par l'augite, la hornblende et la biotite. Cette face très raide s'est effondrée en coulées de blocs au cours de la fonte des glaces ou postérieurement. Le diaclasage est lâche (2 à $4 \mathrm{~m}$ ) et la perméabilité au soluchrome faible, même sur des échantillons superficiels.

Au contraire, la face NE est en pente douce. Elle est éventrée par d'énormes carrières qui montrent la complexité de sa composition. En moyenne, il s'agit de syénites, riches en feldspaths alcalins. Mais l'augite et la néphéline y sont abondantes (souvent 12 à 15\%). Bien qu'il n'existe aucune étude précise à ce sujet, on pourrait soupçonner que la néphéline constitue un élément de faiblesse, tout en n'oubliant pas que certains des reliefs résiduels les plus remarquables du monde sont des foyaïtes (Transvaal, Sintra, Itatiaïa). La composition de ces syénites varie d'ailleurs très rapidement, l'ensemble constituant une brèche gigantesque où la part des éléments ferromagnésiens et des éléments clairs est très différente, ainsi que la teneur en feldspath potassique et en néphéline. Si nous comparons deux échantillons, le plus altéré sur le terrain renferme $60 \%$ des feldspaths alcalins, $12 \%$ de néphéline, $10 \%$ d'augite aegyrinique et $14 \%$ d'aegyrine. Sur l'altérabilité de l'aegyrine, nous ne savons pas non plus grand chose. On remarque seulement que dans ces pyroxènes sodiques le fer est à l'état ferrique, ce qui peut constituer un élément de résistance.

Au total, il semble que la mauvaise tenue du versant soit due à l'extrême fragmentation, en liaison avec l'hétérogénéité mécanique. On trouve des diaclases en moyenne tous les $50 \mathrm{~cm}$. A l'échelle millimétrique également, les fissures sont nombreuses.

L'inselberg du mont Yamaska présente près de 300 $\mathrm{m}$ de dénivellation. II est formé de roches nettement plus basiques. A la partie ENE de la couronne, qui est une des plus raides, correspondent des gabbros. La yamaskite, qui tient son nom de cette intrusion, est composée presque entièrement d'augite titanifère et de hornblende. Elle affleure à la fois dans les alvéoles intérieurs et dans la couronne méridionale. On ne peut la considérer comme un élément de faiblesse. Enfin la façade occidentale est formée en grande partie de cornéennes de métamorphisme de contact et de roches monzonitiques où les plagioclases et les feldspaths alcalins sont en proportion identique. La composition minéralogique joue donc un rôle peu important.

Le complexe de Shefford atteint son maximum d'altitude dans la partie ouest de la couronne où des fragments de trachyte sont emballés dans des porphyres. C'est la texture qui semble responsable de la dureté, et non pas la composition chimique qui est celle de roches intermédiaires. Plus à l'ouest, des syénites renfermant une faible proportion de néphéline (pulaskites) semblent moins résitantes (couloir du lac Coupland et brèche par où passe la route au sud-ouest). Au nord et au sud d'un affleurement central de gabbros, des syénites quartzifères apparaissent relativement en saillie, conformément à une échelle de dureté assez commune. Cependant une échine de gabbros compartimente l'alvéole centrale; sa perméabilité au soluchrome est nulle, même sur échantillon superficiel.

Enfin le complexe de Brome est démantelé en reliefs beaucoup plus modestes, dont la répartition est assez peu conforme à celle que l'on pourrait attendre d'après la carte pétrographique. La plus grande partie de la dépression centrale se trouve dans les pulaskites. II est impossible d'imaginer que ces syénites ont cédé à l'altération, parce qu'elles renfermaient un peu de néphéline (moins de $5 \%$ ). Ce dernier minéral était en effet encore plus abondant dans la calotte du mont Brome (s.s.) qui est formé de foyaïte. II semble d'après les observations que l'aire déprimée soit fortement fissurée, tant à l'échelle des diaclases qu'à l'échelle millimétrique.

Les reliefs périphériques sont formés de roches assez diverses. A l'ouest, le mont Gale est en syénite (mais avec beaucoup de biotite). La paroi sud montre des gabbros qui, toutefois, s'enrichissent vers le haut en feldspaths potassiques, gage de résistance. À l'est, Pyne Mountain est en micropulaskites. La syénite, de texture normale, située plus au nord, ne donne que des croupes molles.

Au total, la mise en saillie de ces différentes roches intrusives par rapport à des schisto-calcaires ne pose pas de problèmes particuliers. L'érosion différentielle ne se manifeste qu'à l'intérieur des structures annulaires. Nous avons vu qu'elle n'est pas toujours conforme au degré d'acidité de la roche. II se peut que l'altérabilité liée à la microfissuration n'ait pas joué un rôle très important. Beaucoup des petites cuvettes internes des structures annulaires peuvent être attribuées à un diaclasage plus important, que seuls les glaciers ont exploités.

\section{B. ESTRIE ET NORD-EST DU VERMONT (coupe 5, fig. 1)}

Cette unité structurale comporte une série métamorphique principalement pélitique, où les sédiments ont été transformés sous des températures modérées (stade régional de la biotite ou du grenat), ne se relevant qu'au voisinage d'un axe orienté SSW-NNE dans le Vermont, où apparaissent des corps intrusifs discordants sur la plus grande partie de leur périphérie; ils ont été mis en place au Dévonien supérieur. De la différence de température résulte un anneau de métaphormisme de contact bien marqué où figure en particulier la sillimanite. 

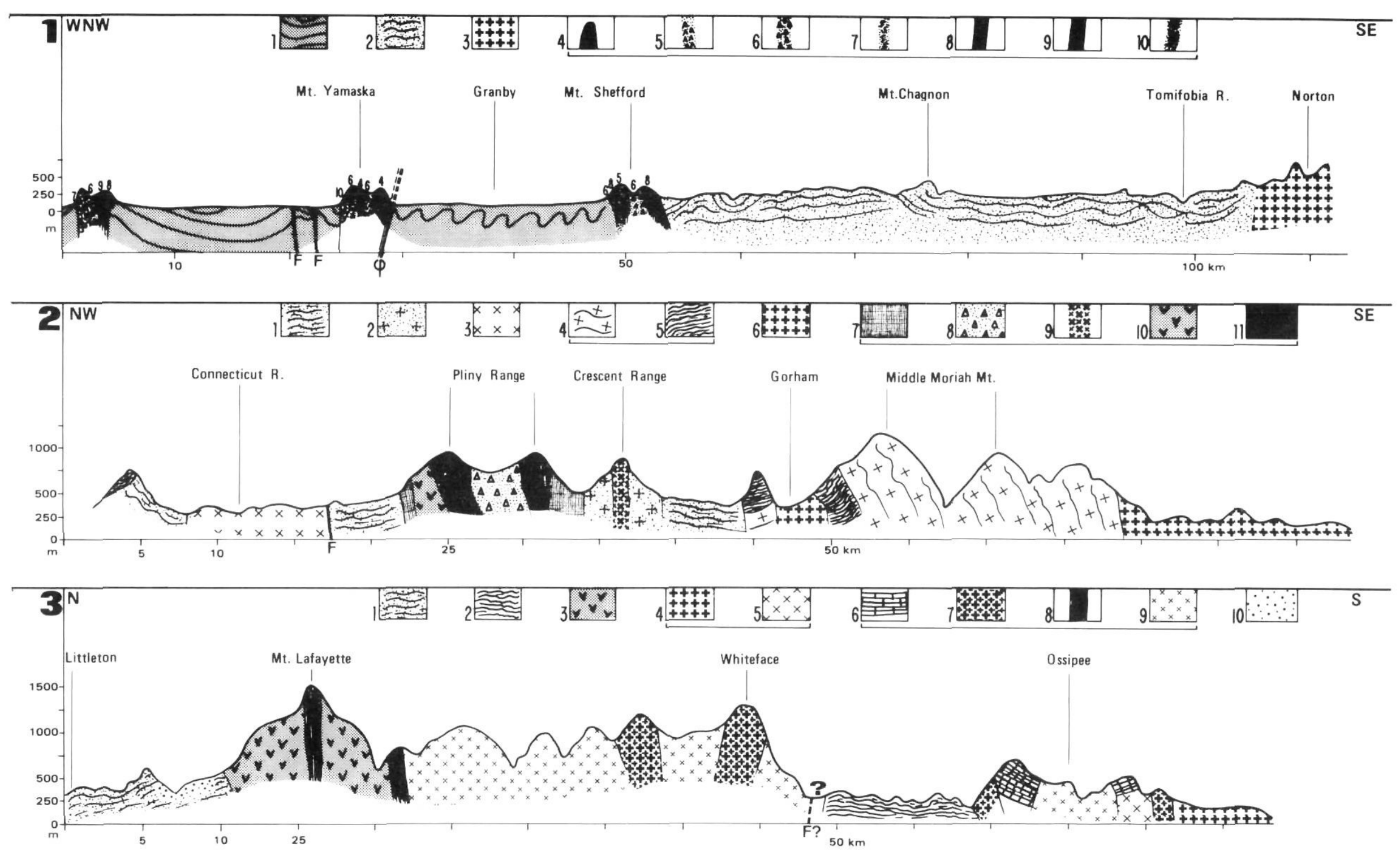

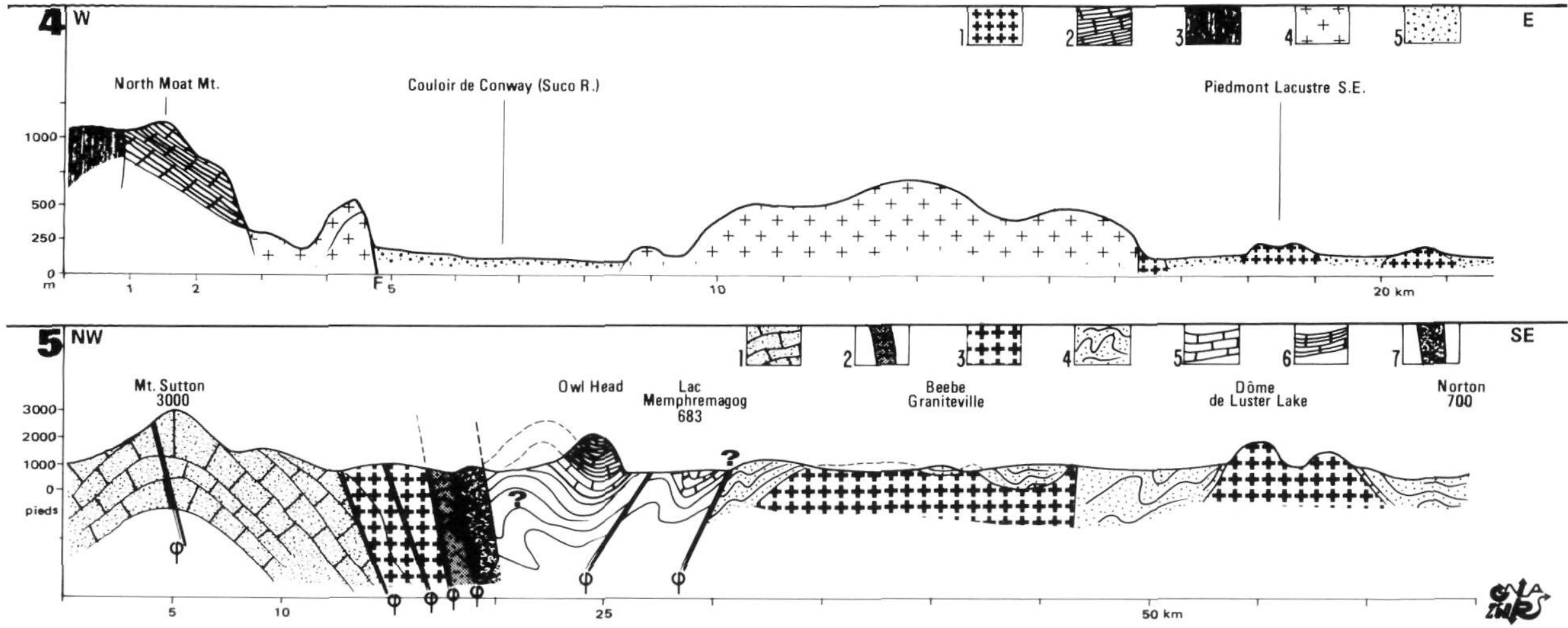

FIGURE 1. Coupes à travers la région étudiée.

Coupe 1: 1) Argilites et calcaires ordoviciens - 2) Cambro-Dévonien métamorphique - 3) Granite dévonien - 4) Yamaskite; - 5) Brèche volcanique à matrice gabbroïque - 6) Gabbro-diorite - 7) Essexite - 8) Syénite - 9) Brèche volcanique à matrice syénitique -10 ) Cornéenne de contact $-\varphi$ : accident chevauchant $-F$ : faille.

Coupe 2: 1) Formation métamorphique ordovicienne -2) Orthogneiss ordoviciens plus ou moins foliés - 3) Granodiorites ordoviciennes - 4) Gneiss de base des séries de Littleton - 5) Schistes des séries de Littleton (avec micaschistes et quartzites) - 6) Granites dèvoniens -7 à 11: Intrusions mésozoïques des White Mountains Series : 7) Syénite grossièrement feuilletée - 8) Diorite - 9) Granite porphyrique -10) Monzonite quartzifère -11) Syénite à hastingsite

Cope 3: 1) Série métamorphique des formations olivériennes - 2) Schistes des séries de Littleton - 3) Monzonite quartzifère de Kinsman - 4) Granite dévonien - 5) Granodiorites dévoniennes - 6 à 9: Intrusions mésozoïques et laves du complexe annulaire: 6) Laves (basaltes à rhyolite) - 7) Granite à riébeckite ou hastingsite - 8) Syénites - 9) Granite de Conway - 10) Formations fluvioglaciaires. Coupe 4: 1) Granite dévonien -2) Laves recuites des séries de Moat - 3) Syénite -4) Granite de Conway - 5) Formations fluvio-glaciaires.

Coupe 5: 1) Schistes, quartzites injectés par des filons de quartz (cambriens?) 2) Quartzites et schistes ardoisiers cambriens - 3) Granite dévonien - 4) Quartzies, slates et calcaires impurs du groupe de Saint-Francois (ordovicien) - 5) Calcaires et slates du groupe de Glenbrooke (silurien-dévonien) - 6) Basaltes, andésites dévoniennes du groupe de Bolton -7 ) Filons de péridotite $-\varphi$ : chevauchement.
Geological cross-sections.

Section 1: 1) Ordovician limestones shales - 2) Cambro-devonian metamorphic series - 3) Devonian granite - 4) Yamaskite - 5) Volcanic breccia with gabbroic matrix 6) Gabbro-diorite - 7) Essexite - 8) Syenite - 9) Volcanic breccia with syenitic matrix - 10) Hornfels - $\varphi$ : thrust, overlap fault $-F$ : Fault.

Section 2: 1) Ordovician metamorphic series -2) Ordovician orthogneiss (more or less foliated) - 3) Ordovician granodiorites - 4) Basement gneiss of the Littleton Series - 5) Littleton Series (with micaschists and quartzites) - 6) Devonian granites - 7 to 11: Mesozoic intrusives of White Mountain Series: 7) Coarse foliated syenite series - 8) Diorites - 9) Porphyric granites - 10) Quartz monzonite - 11) Hastingsite syenite.

Section 3: 1) Metamorphic oliverian formation - 2) Littleton Series - 3) Kinsman quartzmonzonites - 4) Devonian granites - 5) Devonian granodiorites - 6 to 9 : Mesozoic intrusives and ring complex-lavas - 6) Lavas (from basalts to rhyolites) - 7) Riebeckite or hastingsite granites - 8) Syenites - 9) Conway granites - 10) Fluvioglacial deposits.

Section 4 : 1) Devonian granites - 2) Recrystallized lavas of Moat Series - 3) Syenite -4) Conway granites - 5) Fluvioglacial deposits.

Section 5: Schist and quartzites, with quartz veins (Cambrian?) - 2) Cambrian quartzites and slates - 3) Devonian granite - 4) Quartzites, slates and impure limestones of the Saint-François group (Ordovician) - 5) Limestones and slates of the Glenbrooke group (Silurian-Devonian) -6) Devonian basalts and andesites (Bolton group) - 1) Peridotites veins $-\varphi$ : thrust 
Tant dans le SE du Québec que dans le NE du Vermont, le cœur des intrusions a souvent été mis en creux. Dans certains cas, il n'existe pas de bourrelet annulaire continu; les bosses se disposent de façon irrégulière sur la bordure de l'affleurement. Enfin certains corps granitiques étroits sont mis en saillie. D'ailleurs, il ne faut pas oublier que l'érosion glaciaire est responsable de nombreux ombilics, dont l'originalité géologique peut se réduire à une densité plus grande des diaclases.

Les croupes allongées, taillées dans le matériel pélitique en général de grain fin, ne prennent quelque vigueur que là où affleurent des quartzites. Leur altitude et leur degré de dissection diminuent rapidement au nord de la frontière internationale, pour donner un paysage de "pénéplaine".

Le corps granitique de Nulhegan River (Island Pond) présente l'aspect classique en chaudron, creusé de près de $300 \mathrm{~m}$ par rapport aux croupes périphériques. La composition minéralogique peut l'expliquer: il s'agit surtout de granodiorites peu diaclasées, riches en hornblende et biotite. La perméabilité est cependant particulièrement faible (Ko Berneix inférieur à 10-13), les reliefs périphériques correspondent tantôt à des cornéennes, ou à des schistes ayant subi une recristallisation moins complète et assez fortement divisés (cloison SW et NW) - tantôt à des granodiorites dont les cristaux de plagioclase et de biotite avaient été fortement fissurés, puis envahis par de grands feldspaths $K$, trois fois plus abondants que les plagioclases (fig. 2).

Les dômes et demi-dômes à dalles d'exfoliation qui dominent le lac Lyster sont les éléments saillants de la bordure de la grande intrusion granitique NortonCoaticook. Toutes les conditions sont réunies pour rendre compte de cette position dominante. II s'agit d'une roche où les feldspaths potassiques forment souvent plus de $60 \%$ de l'échantillon: ces microclines

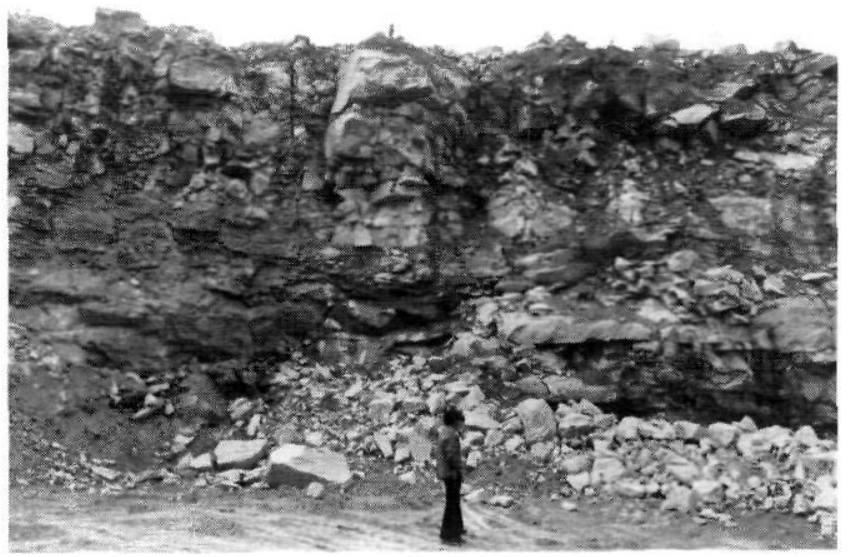

FIGURE 2. Fracturation inégale des granodiorites au fond de la cuvette de Nulhegan River (Island Pond, Vermont). Les altérites ont été nettoyées par le passage des glaciers.

Differential fracturing of the granodiorites at the bottom of the Nulhegan River basin (Island Pond, Vermont). Weathered rocks were carried off by glaciers. tardifs englobent des plagioclases et quelques biotites à fissures assez nombreuses mais scellées par la recristallisation. Même les échantillons superficiels sont complètement imperméables au soluchrome; et sur les parois raides, les diaclases sont très espacées. Dans les dépressions voisines, occupées partiellement par le lac Lyster et le lac Nortor (fig. 3) la composition minéralogique varie rapidement entre des granites analogues à ceux des dômes (parfois $65 \%$ de microclines) et des granites plus sombres et plus riches en plagioclases. La microfissuration visible au microscope et appréciée par le test au soluchrome varie aussi entre $0 \mathrm{~mm} / 15 \mathrm{~min}$. et $10 \mathrm{~mm} / 1 \mathrm{~min}$. ; cependant, dans ce dernier cas, il ne faut pas exclure que la roche ait subi un commencement d'altération. Sous le microscope, sur une même lame prélevée dans la variété riche en microclines, on trouve des plages fissurées alternant avec des plages intactes. L'impression est que les alvéoles, de petite taille, ont été excavés dans des volumes assez hétérogènes, mais avec des diaclases verticales rapprochées.

Le contexte pétrographique général est celui d'un métasomatisme potassique affectant des granites plus basiques anciens. Sous des climats variés, ils se prêtent bien à la formation de dômes (Sierra Leone, d'après MARMÖ (1971) - Transvaal septentrional, etc.). L'hypothèse du métasomatisme paraît s'imposer, puisqu'on trouve au sein de grosses enclaves basiques des porphyroblastes idiomorphes de microclines.

En raison de la diminution de l'énergie du relief vers le nord, le bourrelet qui entoure l'alvéole de Scotstown est plus modeste. Mais, grâce à l'installation d'une vaste carrière au cœur de la dépression, on a la chance de pouvoir étudier en détail sa composition minéralogique. II s'agit d'un granite proche d'une

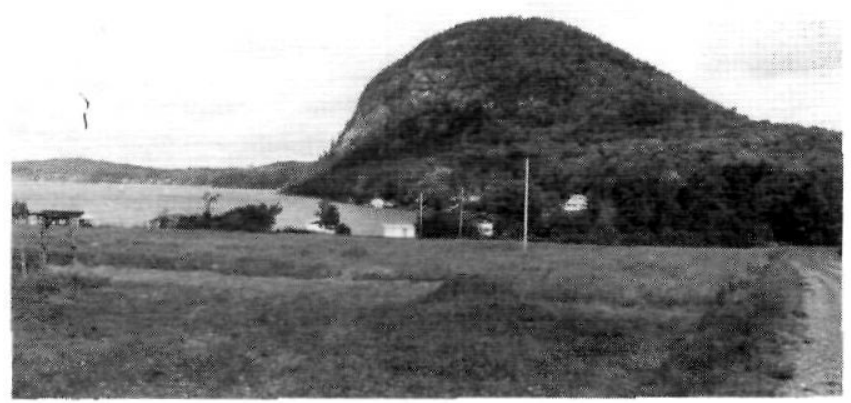

FIGURE 3. En bordure de l'intrusion de Norton-Coaticook, Barnston Pinacle offre son profil dissymétrique dont le flanc raide domine le lac Lyster (Cantons-de-l'Est, Québec). Vue vers le nord-ouest.

Along the Norton-Coaticook intrusion, Barnston Pinacle presents a dissymmetrical profile with its steep side overlooking Lyster Lake (Eastern Townships, Québec). View from the southeast. 
monzonite, mais avec un étage subhorizontal supérieur plus leucocrate, et aussi plus compact que l'étage inférieur. Ce dernier est extraordinairement fissuré; pour cette raison, il est exploité pour la production du ballast. Dans des échantllons parfaitement frais, la pénétration du soluchrome atteint $10 \mathrm{~mm} / 1 \mathrm{~min}$., ceci dans tous les échantillons testés.

La composition minéralogique du bourrelet est très variable. Au NE, où les versants sont particulièrement raides, il se distingue par une richesse plus grande en microcline, ou par la fusion de filons d'aplite. Ailleurs, il est composé de schistes gréseux recristallisés.

Moins continues, encore, sont les auréoles de bordure des intrusions granitiques de Stratford Centre. Celle du nord-est (lac aux îles) est remarquable cependant par le dispositif régulier de la couronne en saillie enveloppant un anneau de granite déprimé et un volume central légèrement plus saillant. Le granite à biotite, qui affleure au centre sous forme de dos de baleine, est particulièrement massif. Sa composition est assez proche de celle du centre de l'alvéole de Scotstown. La partie SE du bourrelet correspond à l'auréole de contact finement recristallisée. Quant à l'échine qui cerne le corps intrusif au sud-ouest, elle est constituée de schistes et quartzites très divisés.

L'intrusion située plus au SE a un ombilic central entièrement occupé par des marécages. Seules les cloisons nord et ouest sont représentées dans le relief, principalement dans le monolithe du mont Aylmer où affleure un granite franc, plus "acide" que ceux de Scotstown, et dont la perméabilité au soluchrome est nulle. Le caractère le plus remarquable est l'écartement des diaclases. On y connaît des blocs homogènes dont les dimensions atteignent $7 \times 4 \times 3 \mathrm{~m}$.

Enfin, l'expression de l'étroite bande granitique orientée SW-NE, qui émerge des schistes métamorphiques entre les monts Sainte-Cécile et Saint-Sébastien, est entièrement différente. II s'agit d'une voûte, "pseudomont dérivé", débarrassé par l'érosion différentielle de sa couverture. Sur une douzaine de kilomètres de long, elle se décompose en dômes atteignant une hauteur relative de $300 \mathrm{~m}$ par rapport à l'encaissant (fig. 4).

La roche dominante, très massive, est un granite franc à perthite. Bien que d'âge dévonien supérieur, il s'apparente par là aux intrusions mésozoïques. Mais il est à noter que les dômes les plus élevés, situés aux deux extrémités, sont très riches en aplite, dont on connaît la résistance particulière à la météorisation chimique. D'autre part, quelques passées de granodiorites affleurent dans les dômes moins élevés qui se trouvent en position centrale. Mais l'individualisation des bosses ne dépend pas des variations de la composition lithologique, l'ensemble de chacune d'entre elles étant enveloppé par de grandes diaclases courbes, en accord avec le versant.

Les cols intermédiaires correspondent à des fractures orthogonales à la direction générale du chapelet

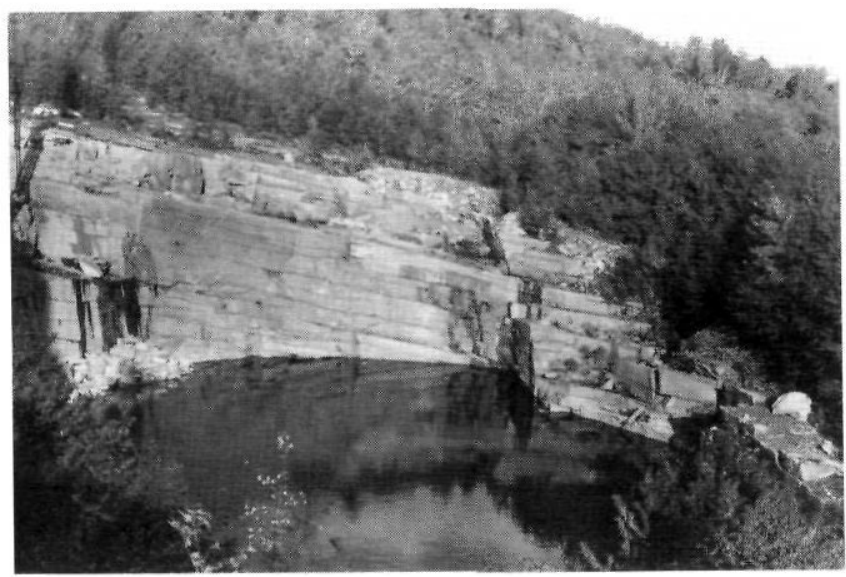

FIGURE 4. Le débit en grandes dalles simples d'exfoliation sur le flanc des dômes de Sainte-Cécile et de Saint-Sébastien (Cantons-de-l'Est, Québec).

Large simple exfoliation slabs on the side of the SainteCécile and Saint-Sébastien domes (Eastern Townships, Québec).

des plis affectant les schistes. A la surface de ces bandes, les diaclases verticales, très espacées sur les dômes, sont plus serrées. D'autre part, l'importance de la microfissuration est très variable. Sur certains blocs, la microporosité au mercure ne dépasse pas $0,15 \%$, tandis qu'un échantillon immédiatement voisin a une porosité à l'eau Berneix de 10-9. Les tests au soluchrome, faciles à réaliser dans de multiples carrières, témoignent de la même hétérogénéité. Le débitage par les diaclases verticales dans ces bandes parallèles à la direction de l'écoulement de la glace du NW au SE a probablement facilité le façonnement des couloirs. Le même réseau orthogonal de fractures exploitées par les vallées et les cols de flanc affecte d'ailleurs toute la région.

Ainsi les relations de dureté avec l'encaissant schisteux sont bien différentes de celles des corps plutoniques excavés en chaudron. Outre la composition minéralogique, très différente de celle de Nulhegan River, il faut rappeler que les schistes sont ici très faiblement métamorphiques, ne dépassant pas le clivage ardoisier. II existe bien une auréole de contact, étudiée par les prospecteurs de molybdène, avec des cornéennes résistant à l'altération chimique. De fait, leurs affleurements montent très haut sur les pentes SW du mont Sainte-Cécile. Et surtout, elles constituent deux bosses mineures à l'extrémité NE du chapelet, où le toit de granite descend très lentement, en envoyant de multiples filons dans l'encaissant.

II est probable que de petites failles longitudinales, SSW-NNE, ont contribué à la surrection du toit de l'intrusion par rapport aux schistes (succédant peutêtre à la déchirure qui a permis l'ascension des granites). Mais bien entendu, on ne peut retenir les idées de RITCHOT (1975) qui considère comme formes structurales primitives des reliefs de ce genre, alors que l'érosion 
différentielle n'a fait que mettre en valeur des structures acquises au Dévonien (tabl. I).

\section{TABLEAU I}

Pourcentage moyen des principaux minéraux

\begin{tabular}{lccccc}
\hline & Q & FA & PI & B & M \\
\hline Granite de Saint-Sébastien & 27 & 33 & 28 & 8,5 & 1 \\
Granite de Scotstown & 32 & 25 & 28 & 13 & 2 \\
$\begin{array}{l}\text { Cœur de la dépression } \\
\text { du lac aux Îles }\end{array}$ & 34 & 20 & 28 & 15 & 2 \\
\hline
\end{tabular}

$\mathrm{Q}$, quartz; FA, feldspaths acalins; PI, plagioclases; B, biotite; $M$, muscovite.

Le complexe intrusif du mont Mégantic (CLÉMENT et PELLETIER, 1980). Avec un profil de pseudo-Vésuve, le croissant et le dôme central du mont Megantic dominent de $500 \mathrm{~m}$ la "pénéplaine" développée dans les pélites. En dépit de la discontinuité des affleurements, les relations entre le relief et la composition minéralogique de l'intrusion apparaissent simples et logiques.

Un croissant de syénite porte les points culminants. Il est séparé d'une grosse bosse granitique par une dépression hémicirculaire taillée dans les gabbrodiorites. La résistance à l'érosion différentielle est fonction de l'abondance des feldspaths alcalins (perthite) et du quartz.

On observe que la biotite du granite, peu abondante, est très dispersée et que les syénites bénéficient d'une architecture engrenée (tabl. II).

\section{TABLEAU ॥}

Complexe intrusif du mont Mégantic (d'après REID, 1976)

(minéraux en pourcentage)

\begin{tabular}{lcccccc}
\hline & FA & PI & Q & B & Am & Py \\
\hline Syénite & 75 & 16 & 4 & & 2,6 & 1,4 \\
Gabbro-diorite & & 63 & & 2,3 & 17 & 9,6 \\
Granite & 43 & 23 & 29 & 3,8 & & \\
\hline
\end{tabular}

Q, quartz; FA, Feldspaths alcalins; PI, plagioclases; B, biotite ; Am, amphibole, Py, pyroxène.

Mais on notera un certain parallélisme avec les données concernant la porosité-perméabilité. L'examen microscopique de la syénite du croissant, au sommet portant l'antenne de radio, montre que les cristaux sont fissurés mais bouchés par l'oxyde de fer. Les échantillons superficiels des flancs sont complètement imperméables au soluchrome. Quant à la bosse du granite, les échantillons superficiels ont une perméabilité Berneix inférieure à $10^{-12}$, une microporosité au mercure de $0,05 \%$, et sont complètement imperméables au soluchrome.
II est plus difficile de trouver des gabbros sains, puisqu'ils s'altèrent en une arène à nontronite, située sous la moraine (CLÉMENT et DE KIMPE, 1977). Cependant, elle apparaît à la faveur de rapides, et sa perméabilité au soluchrome est de valeur moyenne.

\section{LES INTRUSIONS SEPTENTRIONALES DU NEW} HAMPSHIRE (fig. 5)

Entre le Connecticut et le massif relativement continu des White Mountains, le relief se présente de façon anarchique, à l'exception de la mise en valeur de complexes intrusifs approximativement annulaires, d'âge plus ancien que celui du mont Mégantic, puisque leur datation se place entre 200 et 170 millions d'années (coupe 2, fig. 1).

La raideur des pentes dépasse rarement en moyenne $25^{\circ}$, se décomposant parfois dans des détails en plusieurs parois rocheuses atteignant quelques mètres de haut, et masquées par la forêt. Dans certains cas au moins, cette discontinuité peut être l'effet de coups de gouge donnés par les glaciers dans une masse irrégulièrement diaclasée.

Les géologues américains qui ont levé la carte entre 1930 et 1950, ont eu, selon la tradition des États-Unis, une claire conscience du rôle de l'érosion différentielle dans le paysage. Sans doute, ce sentiment les a-t-il entraînés à vanter exagérément telle forme structurale considérée comme une des plus pures du monde, et à dessiner certains contours en fonction des courbes de niveau (car dans bien des dépressions, la roche en place n'est objectivement pas visible).

1. Le relief d'érosion différentielle atteint son maximum de vigueur dans le complexe annulaire des Pliny Ranges, mis en saillie par rapport aux formations dites olivériennes.

L'armature de la crête principale en croissant est constituée par des syénites quartzifères renfermant une quantité non négligeable d'hastingsite (surtout par sa signification quant aux conditions de mise en place).

FIGURE 5. Carte morphostructurale des White Mountains (s.I.).

Morphostructural map of the White Mountains (s.l.). From top to bottom: 1) Ordovician Oliverian series (chiefly gneissic) -2) Older and later metamorphic series outcropping below $750 m-3)$ Metamorphic Devonian Littleton Series, chiefly schistose outcropping above $750 \mathrm{~m}-4$ ) Littleton gneissic series, outcropping above $750 m-5)$ Devonian or older granodiorites excavated into basins - 6) Devonian granite position - 7) Protuding Devonian granite - 8) Granites or granodiotites incorporated into the White Mountains - 9) Isolated intrusive of mesozoic granites, in crowning position 10) Mesozoic intrusive and effusive complex, ranging from syenites to rhyolites building up the chief part of the White Mountains - 11) Arcuate syenitic ridges in ring structures 12) Syenitic stocks in crowning position - 13) Hollows in the Conway granites - 14) Main fractures controlling furrows and gorges - 15) Marginal scarps of the White Mountains. 


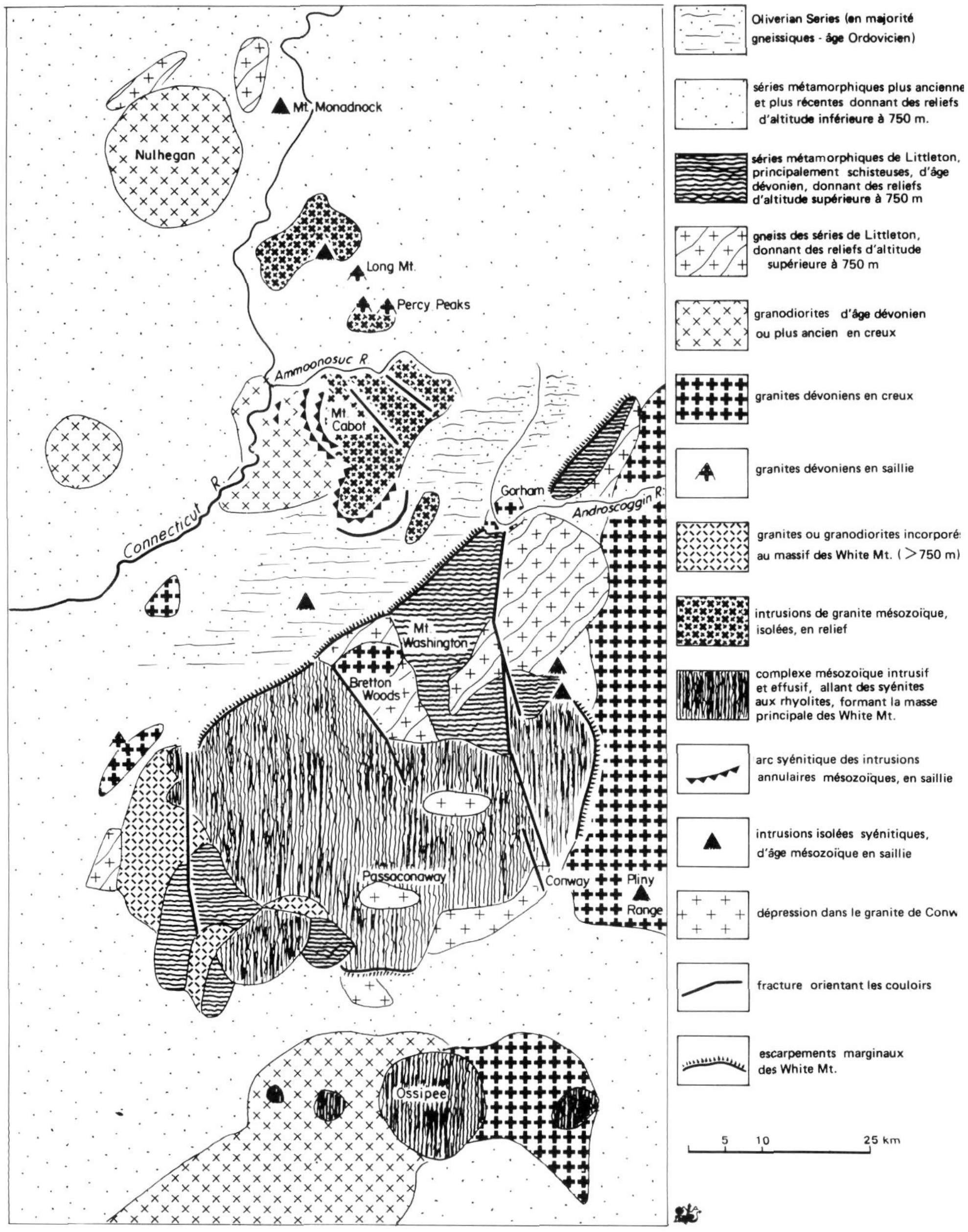


Le fait essentiel est que la somme des minéraux peu altérables (quartz + feldspaths alcalins) atteint de 70 à $90 \%$ du volume. En position relativement externe, affleure localement une syénite à grain moyen donnant les contreforts rocheux du versant sud des Pliny Ranges (s.s.). La somme des minéraux non altérables y est encore très importante, surtout dans la petite unité orographique envisagée.

La syénite à grain grossier donne des pentes de valeur moyenne sur le versant est, et aussi une partie de la dépression périphérique la séparant d'un croissant tout à fait externe, Crescent Range. Elle avait été rattachée dans les cartes précédentes aux unités olivériennes. Elle est, dans l'ensemble, plus riche en plagioclases. Sa position basse dans le sillon périphérique qui la sépare de Crescent Range s'explique peut-être par la présence d'une zone de fissuration, provoquée par l'ascension du bouchon du magma central; ainsi se justifierait la légère foliation qui l'apparente aux séries olivériennes. L'individualisation de Crescent Range pourrait alors être attribuée à la fois à l'exploitation de ces anneaux fracturés, et à l'intrusion d'un granite porphyrique dans les séries olivériennes riches en feldspath alcalin. Malheureusement, la carte moderne ne représente qu'une partie de ce relief, et nous n'avons pas eu l'occasion d'échantillonner dans cette région.

Ce croissant se prolonge par un dyke de composition analogue, constituant l'anneau le plus externe des Pliny Ranges. Les échantillons prélevés dans la région de Jefferson nous ont montré qu'il est intensément fracturé, ce qui serait en bon accord avec l'hypothèse précédente. Cependant, l'étude des xénolithes, faite par CZAMANSKE et al. (1977) a montré qu'ils sont analogues en tous points aux roches de l'Olivérien encaissant, ce qui impliquerait que le frottement ne s'est pas accompagné d'un grand déplacement vertical.

Toutefois, sur le versant SW, des contreforts à versants raides ne semblent pas seulement soutenus par la roche dure de la crête de syénites quartzifères majeure; or, ils sont taillés dans des ring-dykes composés de granitoïdes plus pauvres en feldspaths alcalins et en quartz (la somme de ces deux éléments étant souvent inférieure à $50 \%$ ). Leur mise en saillie par rapport aux gneiss à hornblende des formations olivériennes de la région de Jefferson ne paraît plus explicable par la composition minéralogique.

Le cœur des intrusions est occupé par la diorite, mise en creux (autant qu'on puisse en juger sous une couverture détritique), par rapport à l'arc syénitique. Mais, assez paradoxalement, elles forment le Mount Weeks, un des plus hauts sommets du croissant principal, en s'adossant à la syénite (tabl. III).

2. L'ensemble de ces intrusions est mis en saillie par rapport aux formations que la carte géologique désigne sous le nom d'Oliverian Series: un complexe de gneiss et de granitoïdes faiblement foliés. Elles donnent
TABLEAU III

Pourcentage de minéraux peu altérables (d'après les analyses de CZAMANSKE et al. 1977)

\begin{tabular}{|c|c|c|c|c|c|c|c|c|c|c|c|c|c|}
\hline \multicolumn{2}{|c|}{ H.Q.S. } & \multicolumn{2}{|c|}{ M.G.S. } & \multicolumn{2}{|c|}{ C.S. } & \multicolumn{2}{|c|}{ D. } & \multicolumn{2}{|c|}{ Q.M.D. } & \multicolumn{2}{|c|}{ P.Q.M. } & \multicolumn{2}{|c|}{ G.C. } \\
\hline Q & $\mathrm{FA}$ & $Q$ & FA & Q & FA & Q & FA & Q & FA & Q & FA & $Q$ & FA \\
\hline 20 & 52 & 6 & 74 & 2 & 64 & 1 & & 5 & 12 & 24 & 27 & 40 & 50 \\
\hline 15 & 65 & 8 & 63 & 1 & 80 & & & 5 & 26 & 24 & 34 & & \\
\hline 16 & 57 & 8 & 67 & 4 & 63 & & & 9 & 12 & & & & \\
\hline 18 & 73 & $\times 7$ & $\times 41$ & 7 & 58 & & & 7 & 1 & & & & \\
\hline & & $\times 22$ & $\times 44$ & 1 & 67 & & & & & & & & \\
\hline & & & & 9 & 56 & & & & & & & & \\
\hline & & & & 14 & 47 & & & & & & & & \\
\hline & & & & & 43 & & & & & & & & \\
\hline
\end{tabular}

H.Q.S., syénites à quartz et hastingsite; M.G.S., syénites à grain moyen ( $\times$, échantillon prélevé en dehors des Pliny Mountains) ; C.S., syénite à grain grossier; D., diorite; Q.M.D., diorite monzonitique quartzifère; - P.Q.M., monzonite quartzifère; G.C., granite de Crescent Range, Q, quartz ; FA, feldspaths alcalins.

d'ailleurs lieu à un relief assez varié, avec tantôt des alvéoles assez larges, tantôt de grosses bosses.

Les auteurs ne sont pas d'accord sur l'importance relative des paragneiss et des orthogneiss.

La composition minéralogique des gneiss est très variable, depuis des roches peu "acides", riches en hornblende ou biotite et en plagioclases ( $33 \%$ de An), jusqu'à des gneiss ayant la composition d'un granite franc (Q: $31 \%-F K: 34 \%-P I$ : (An 17) $32 \%$ B : $2 \%$ ). II ne semble pas que ces variations aient des répercussions topographiques systématiques; les flancs de l'alvéole de Jefferson, dont le fond rocheux est masqué, se trouvent tantôt dans les gneiss à hornblende, et tantôt dans des granites légèrement feuilletés.

II est vrai que les basses collines entre Riverton et White Field arasent les roches de composition granodioritique; mais elles se trouvent à proximité du niveau de base le plus "avantageux" de la région, c'est-à-dire le Connecticut. Le relief est plus élevé, avec des pentes plus raides entre les contreforts de Crescent Range jusqu'au delà de Berlin. Dans le secteur SW, il s'agit de granites à foliation, donc en principe plus résistants à l'altération chimique. Mais, dans le secteur de Berlin, si l'on excepte les dômes (fig. 6), les gneiss à deux micas sont plus riches en plagioclases. Plus au nord encore, où les versants s'écartent, leur composition devient franchement granodioritique.

II ne faudrait pas accorder trop d'importance à cette confrontation. En effet, on passe de la "granodiorite" au "granite», principalement par augmentation du nombre des phénocristaux roses de feldspaths potassiques. Mais ces derniers n'arrivent jamais à se toucher, si bien que la résistance dépend de celle de la matrice. 


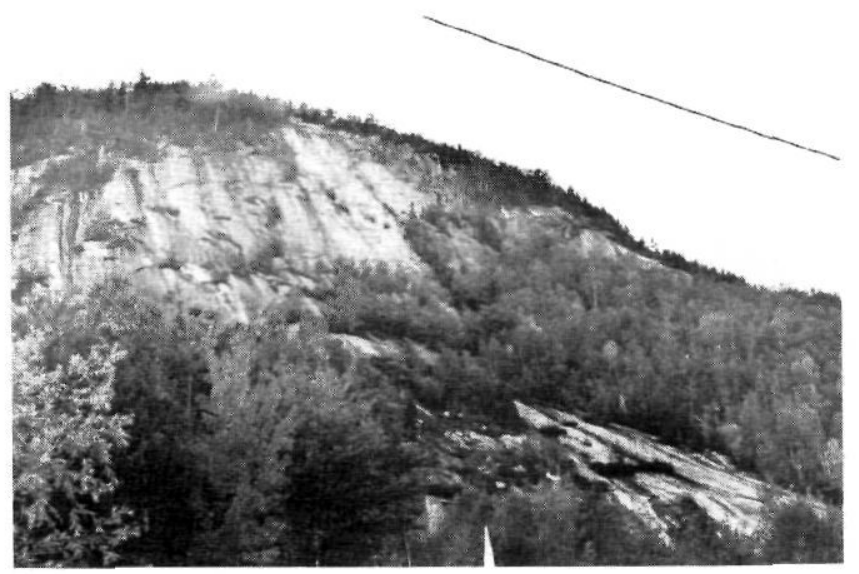

FIGURE 6. Le flanc est du dôme de Mount Forest, au-dessus de Berlin (New Hampshire): dans des monzonites très massives à quartz orientés, injection de puissantes veines plissotées de pegmatites.

On the eastern side of Mount Forest dome, above Berlin (New Hampshire), powerful injection of crumpled veins of pegmatite within very massive monzonites with oriented quartz.

Le point commun à tous les échantillons examinés dans la dépression relative située entre Berlin, Randolf et Jefferson est que les feldspaths, et aussi les éléments ferromagnésiens, portent des traces d'écrasement, suivi d'une recristallisation incomplète. On notera leur forte perméabilité au soluchrome (bien que la valeur de cette observation soit diminuée par la rareté des carrières). Ces faits sont à rapprocher de l'étude de PAGÉ (1968) sur des séries olivériennes situées dans les dômes en bordure du Connecticut: les granites (s.l.) montrent la surimposition d'une action cataclastique à l'esquisse de foliation originelle, la recristallisation ultérieure restant incomplète. Les feldspaths primitifs et les quartz sont amenuisés en grains, et les biotites tordues.

Notons que la trituration tectonique affecte non seulement le fond monzonitique orienté, mais souvent aussi les bandes de pegmatites plus tardives. Seuls des filons basiques postérieurs y échappent.

On peut donc admettre l'hypothèse que le métamorphisme d'âge dévonien tardif, qui a affecté tout l'ensemble de la région, n'a été suivi que d'une recristallisation incomplète, d'où une perméabilité notable des roches. II se peut qu'une fracturation encore plus tardive (d'origine thermique?) ait affecté des croissants parallèles aux intrusions du Mésozoïque inférieur des Pliny et Crescent Ranges, déterminant les dépressions principales de la région.

Exceptionnellement, sont inclus dans la même formation géologique un chapelet de dômes rocheux (SW de Berlin) (fig. 6). Les gneiss y sont remplacés soit par une association mésocristalline de quartz (jusqu'à $60 \%$ ) et d'albite, soit par une pegmatite intensément plissée et recristallisée, où la proportion de quartz est aussi très forte. Même pour les échantillons superfi- ciels, la perméabilité au soluchrome est nulle. Les diaclases courbes limitant les dômes recoupent indifféremment les deux formations.

3. Les intrusions plus septentrionales ont une disposition géométrique, et aussi une expression orographique moins simples.

Le relief d'érosion différentielle atteint son maximum de vigueur dans le secteur sud-ouest où a été mis en creux le vieux corps plutonique de Lost Nation, qui remonte peut-être jusqu'à l'Ordovicien supérieur, d'où une foliation localement assez accentuée. La composition minéralogique est très variable, depuis des diorites faiblement quartziques où domine l'andésine, puis la hornblende verte $(30 \%$ de la roche totale), jusqu'à de véritables granites à deux micas, avec des feldspaths potassiques perthitiques ou microcliniques; ils englobent les petits cristaux résiduels à cœur séricitisé de biotite et de quartz, et résultent sans doute d'un métasomatisme tardif, succédant à un premier épisode de fissuration et de rétromorphose, avec calcite et épidote. Cependant, deux facteurs conjugent leurs effets pour rendre compte de la faible résistance à l'érosion: a) en moyenne dominent les faciès proches des diorites ou des granodiorites; b) les phénocristaux potassiques n'ont pas réussi à cicatriser toutes les fractures de la première génération, et ils sont eux-mêmes fissurés. Tout se passe comme si les effets de l'orogénie acadienne n'avaient pas été suivis d'une recristallisation suffisante (tabl. IV).

\section{TABLEAU IV}

Composition moyenne des intrusions septentrionales en pourcentage (d'après CHAPMAN et RABBIT, 1947 et nos échantillons)

\begin{tabular}{lrrlrr}
\hline & Q & FA & PI & B & Am \\
\hline Syénites de Pilot Range & 2 & 82 & 0 & & 12 \\
Granite à hastingsite & 24 & 66 & 0,5 & & 8 \\
$\begin{array}{l}\text { Granite à riébeckite } \\
\text { Granite de Conway à }\end{array}$ & 38 & & & & \\
grain grossier & 34 & 44 & 17 & 5 & \\
$\begin{array}{l}\text { Granite de Conway } \\
\text { à grain moyen }\end{array}$ & 32 & 37 & 26 & 2 & \\
\hline
\end{tabular}

Q, Quartz; FA, feldspaths alcalins; PI, plagioclases; B, biotite ; A., amphibole.

Au-dessus de cette dépression s'élèvent les parois syénitiques de Pilot Range, flanquées du croissant plus modeste dont le toponyme est expressif: Cape Horn. Si la pente d'ensemble du versant est relativement douce, elle est accidentée par une série de dalles composées de syénites, qui renferment jusqu'à $80 \%$ de microperthites. La pénétration du colorant y est nulle. Les petits replats entre les dalles semblent correspondre à des zones broyées, avec infiltration hydrothermales. Lorsque les affleurements sont visibles, il s'agit 
d'un granite à microcline tardif englobant des fragments résiduels de plagioclases. La présence d'épidote et calcite atteste une évolution régressive sous influence hydrothermale. les microclines les plus tardifs sont eux-mêmes fissurés. Ceci montre que les derniers épisodes de l'ascension de l'intrusion annulaire se sont accompagnés de frottements dont les fissures ont été incomplètement bouchées.

A l'est et au nord, la géométrie des intrusions est moins simple et s'exprime de façon moins efficace dans le paysage.

Sans doute, elles dominent dans l'ensemble les formations olivériennes et les séries métamorphiques d'Albee, alternances de schistes, de micaschistes et de quartzites en lits minces, d'âge ordovicien. Mais les orientations principales du relief paraissent suivre des fractures et des filons orientés respectivement WSW-ENE et NNW-SSE; elles recoupent indifféremment les intrusions et les roches métamorphiques, en guidant des alignements de bosses. Elles ont été exploitées par les courants glaciaires (South Pond, etc.), en fonction du degré de diaclasage plus que de la composition minéralogique.

Le granite des intrusions revêt des faciès assez divers : faciès hypersolvus à hastingsite particulièrement robuste dans le verrou de Devil Slide - faciès à deux temps de cristallisation, avec une trame de petites orthoses, quartz et biotite englobés par de grands felspaths potassiques qui scellent toutes les fissures antérieures (Beach Hill) et versant est de Pilot Range. Parfois, dans un faciès analogue, les fissures affectent encore les feldspaths potassiques tardifs.

Un autre élément de diversité est le rapport perthite/plagioclases qui s'abaisse pour les variétés les plus arénisables, jusqu'à une valeur de 2-3.

C'est le premier faciès qui est le plus imperméable aux colorants. Très proches de lui sont les granites à riébeckite qui culminent au Mount Cabot.

Enfin, à l'extrémité septentrionale, ce sont des granites dévoniens qui se trouvent en position culminante à Long Mountain, entre 1000 et $1100 \mathrm{~m}$. Cela s'explique par leur extrême richesse en feldspaths potassiques. Sans doute même les microclines tardifs sont affectés de fissures, mais ils ont été scellés par des oxydes de fer. Selon la carte géologique, la même roche s'étendrait à une cuvette lacustre plus septentrionale. L'abondance des moraines a interdit toute vérification de ces propriétés.

\section{LE MASSIF DES WHITE MOUNTAINS (S.L.) ET SON PIEDMONT S.E.}

S'opposant au morcellement général de la topographie, les White Mountains (s.I.) s'allongent du SW au NE, en formant un massif relativement continu dépassant $1000 \mathrm{~m}$ sur plus de $60 \mathrm{~km}$ (fig. 5).
Bien que servant de ligne de partage des eaux entre les rivières qui ont un accès direct à l'Océan, et celles qui sont subordonnées au Connecticut, dans la plus grande partie de la carte, ce massif n'est pas un relief résiduel de position. D'ailleurs, au moins à son extrémité NE, il est traversé de part en part par l'Androscoggin River, qui s'inscrit dans l'ensellement topographique assez large, ce qui est peu favorable à l'hypothèse d'un tracé récent résultant du barrage par l'inlandsis.

La question qui se pose est de savoir si l'érosion différentielle suffit à justifier l'importance du relief, ou s'il ne faut pas y ajouter l'héritage d'une tectonique de blocs (coupes 3 et 4 , fig. 1).

1. La limite nord de la partie orientale du massif, à laquelle on peut donner le toponyme du Mount Washington, correspond bien au contact entre les formations olivériennes et la série métamorphique principalement schisteuse, dite de Littleton, à laquelle, dès ses premiers levés, BILLINGS (1937) a reconnu une résistance exceptionnelle.

Si l'escarpement qui limite cette formation vers le NW est rectiligne, c'est parce qu'une faille très ancienne met en contact avec les séries de Littleton la formation olivérienne ou les schistes de la série Ammonoosuc. Cette faille avait fonctionné en abaissant vers le SE les séries de Littleton du Dévonien inférieur, dont le métamorphisme acquis pendant l'orogenèse acadienne est beaucoup plus poussé que celui du domaine situé au NW.

Mais pourquoi le produit de ce métamorphisme a-t-il donné des roches plus résistantes dans le compartiment sud-oriental?

Les gneiss à deux micas de la base de la série de Littleton constituent une bonne partie du front du massif; surtout, ils affleurent largement dans les hautes croupes orientales. Pourtant, si l'on considère leur composition minéralogique, rien ne les prédispose à ce rôle. Les éléments altérables, plagioclases et biotite, constituent parfois plus de la moitié de la roche et au total ne diffèrent pas beaucoup de ceux des formations situées au NW. On peut seulement noter qu'ils sont pratiquement imperméables au soluchrome, même pour la plupart des échantillons superficiels (tabl. V).

A côté des quartzites, dont les bancs les plus épais donnent des replats (Mount Chandler), l'élément le plus résistant de la série de Littleton est constitué par une formation très hétérogène se débitant en prismes irréguliers, et non pas en parallélépipèdes, et que BILLINGS (1937) étiquette comme coarse rough pseudoandalusite schists, tout en reconnaissant son rôle directeur dans le relief des sommets de Presidential Range (fig. 7). Macroscopiquement, elle a l'allure d'une pseudo-brèche où interviennent une série de nœuds constitués de grands cristaux de sillimanite et de muscovite, remplaçant des cristaux d'andalousite. Ils sont 
TABLEAU V

Composition modale (en pourcentage) des principaux types de roches du massif du Mount Washington

(d'après BILLINGS, 1937, 1941)

\begin{tabular}{cccccccc} 
& Q & FK & PI & B & M & Si & C + S \\
\hline $\mathrm{A}_{1}$ & $20-40$ & $45-3$ & $10-44$ & $5-12$ & & & \\
$\mathrm{G}$ & 37 & & 36 & 15 & 11 & & \\
$\mathrm{SS}$ & 57 & & & 24 & 12 & & \\
$\mathrm{CS}$ & 23 & & 3 & 20 & 40 & 3 & 8 \\
$\mathrm{Bi}$ & 30 & 38 & 24 & 4 & 4 & & \\
\hline
\end{tabular}

$A_{1}$, gneiss fins provenant du métamorphisme de la série de Ammonoosuc; G, gneiss de base de la série de Littleton; SS, schistes à staurotide de la série de Littleton; CS, schistes grossiers à nœuds de grands cristaux de sillimanite; $\mathrm{Bi}$, Granite de Bickford. Q, Quartz; FK, Feldspaths potassiques; PI, Plagioclases; B, Biotite; M, Muscovite; Si, Sillimanite; C+S, Chlorite + Séricite.

tordus, cassés et fortement soudés à une matrice schisteuse plus fine constituée de lits de quartz, de biotite et de muscovite. La pétrogenèse proposée par BILLINGS (1937) laisse encore le lecteur dans l'embarras. Les grands cristaux d'andalousite primitifs seraient dus à un métamorphisme de contact (en fonction de quelle intrusion?). Alors seraient intervenues les déformations les plus importantes visibles dans les nœuds. Puis l'élaboration des micaschistes, toujours en régime de compression. La transformation de l'andalousite en muscovite et sillimanite implique le franchissement d'un isograde, probablement par l'élévation de la température; celle-ci serait particulièrement importante près de la voûte des anticlinaux (montée de solutions chaudes?), ondulations anticlinales que l'auteur identifie dans les croupes culminantes de Presidential Range (en partie en s'appuyant sur la position de métacarbonates, considérés comme sous-jacents aux séries de Littleton). La dernière étape serait l'intervention d'un discret rétrométamorphisme, d'où l'apparition d'une certaine quantité de chlorite et de séricite: ces minéraux sont présents dans les éléments les plus résistants de la série et ne sont pas un facteur de faiblesse.

Quelle serait l'origine de cette exceptionnelle résistance? Évidemment, il y a prédominance d'éléments résistants à l'altération chimique (surtout quartz et muscovite); mais la proportion n'est pas plus grande que dans d'autres schistes du même massif. Par ailleurs, il faut noter que la multiplication des nœuds interrompt la régularité des plans schisteux, et augmente la résistance à la rupture par compression, en particulier les décollements, qui ont pu se poursuivre assez tardivement lors de l'orogenèse acadienne.

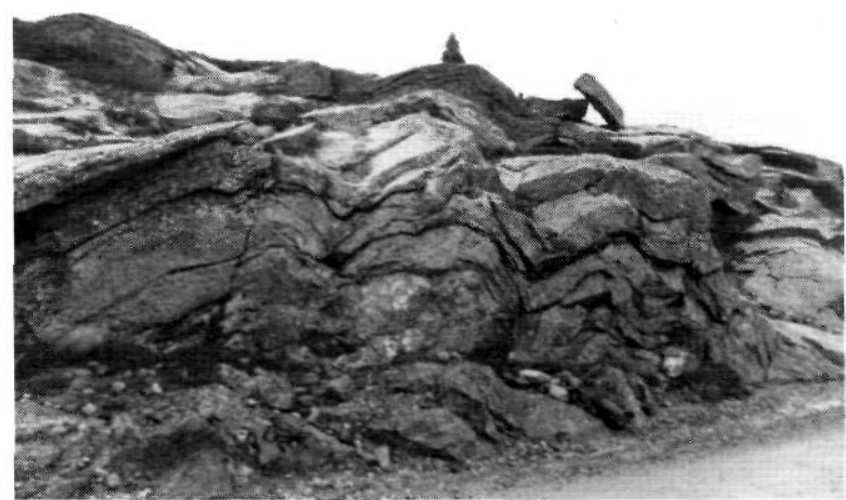

FIGURE 7. Au sommet du Mount Washington (Presidential Range, New Hampshire), le débit en dalles très irrégulières est tributaire de plissotements dans les schistes cristallins.

At the summit of Mount Washington (Presidential Range, New Hampshire), splitting into very irregular slabs is due to crumpling within the crystalline schists.

Quelle que soit la validité des idées exprimées par BILLINGS (1937), il y a plus de 40 ans, on compte deux à trois phases de cristallisation, avec des orientations qui ne sont pas strictement parallèles. Les grands porphyroblastes de muscovite, qui emballent les autres minéraux, en particulier les quartz fissurés, semblent avoir échappé à toute fracturation.

Au sud de l'alvéole de Gorham, la série de Littleton est transformée en migmatites résistantes qui ont fait l'objet d'une analyse approfondie (DOUGAN, 1979) les leucosomes sont constitués principalement de quartz, plagioclase et muscovite, tandis que dans les mélanosomes il s'y ajoute de la biotite et de la sillimanite. Dans l'un et l'autre cas, la cristallisation est postérieure aux mouvements de déformation; cela est évident surtout pour les leucosomes où les muscovites n'ont pas d'orientation privilégiée; et cette proposition s'applique aussi aux mélanosomes où les quartz et les feldspaths présentent des figures de recristallisation de recuit (avec des points triples joignant des angles de $120^{\circ}$ ). II y a là un facteur indéniable de solidité. Cependant, tous les mécanismes mis en place ne sont pas expliqués. L'auteur pense que les leucosomes proviennent de la cristallisation d'un magma local et immobile, enrichi en $\mathrm{SiO}_{2}$ et en $\mathrm{Na}_{2} \mathrm{O}$ provenant de la bordure des mélanosomes. Mais comment cette migration s'estelle produite? Que sont devenues les biotites et la sillimanite situées à l'emplacement des leucosomes? On n'a une réponse satisfaisante à la deuxième question que lorsqu'il existe, dans les leucosomes, des feldspaths potassiques (ou des microperthites) et du grenat. La transformation s'écrit: biotite + sillimanite +2 quartz $=$ feldspath potassique + grenat + eau.

Lorsque cette situation n'est pas réalisée, la biotite a-t-elle été écartée mécaniquement? Enfin, sur les bor- 
dures du mélanosome, quelles sont les cristallisations qui ont comblé les vides laissés par le départ de $\mathrm{SiO}_{2}$ et $\mathrm{Na}_{2} \mathrm{O}$ ?

A l'intérieur du massif du Mount Washington apparaissent des alvéoles (Bretton Woods, Sud de Gorham, etc.) qui correspondent à des affleurements de granite de Bickford. Bien que les roches observées soient souvent massives, elles se comportent comme des roches tendres vis-à-vis des séries de Littleton qu'elles ont pénétrées probablement vers le Dévonien supérieur. Les raisons de cette faible résistance ne sont pas claires. II s'agit d'un granite à deux micas, à structure parfois planaire et où les feldspaths alcalins l'emportent sur les plagioclases (rapport de 2 à 5 ) et où la biotite est disposée en petits nids. En revanche, dans la plupart des tests, la pénétration du colorant est extrêmement rapide. Macrocospiquement, les dislocations sont enregistrées par les filons qui émanent du pluton.

La raideur des cloisons des alvéoles est en liaison avec la recristallisation qui s'est produite au contact avec les gneiss et les schistes de la série de Littleton; elle se manifeste à toutes les échelles, depuis celle des filons périphériques broyés jusqu'à celle des cristaux de quartz qui forment alors jusqu'à $50 \%$ de la roche. Ailleurs le granite lui-même a été laminé et a recristallisé en blastomylonites. Dans la région de Conway, il apparaît aussi que le granite de Bickford est plus tendre que celui des White Mountains Series. Au total, le seul facteur susceptible d'expliquer ce comportement de faiblesse est une microfissuration d'origine tectonique qui, dans le centre des alvéoles, n'aurait pas été suivie de recristallisation, en raison du caractère centrifuge de la circulation hydrothermale ${ }^{1}$.

Le rebord SE du massif, moins élevé et moins rectiligne que celui du NW, correspond approximativement au contact entre les séries de Littleton et un vaste corps de granite dévonien apparenté au granite de Bickford, arasé par une basse plate-forme trouée de lacs. Ces régions basses s'étendent un peu aux dépens des séries de Littleton, là où elles sont constituées par des silicates de calcium provenant du métamorphisme de séries carbonatées.

2. Les hautes croupes de la partie occidentale et méridionale du massif sont taillées principalement dans le plus large des complexes intrusifs du Mésozoïque ancien. II s'y ajoute des enclaves de largeur plurikilométrique des séries de Littleton, en position légèrement déprimée.

L'ensemble des profils évoque le fantôme d'un bloc basculé en direction du SSE et se terminant vers le NNW par un escarpement dominant les collines de la région de Franconia beurrées de dépôts morainiques.

1. Cependant, il faut noter que, dans le centre des cuvettes, la roche en place est couverte par la moraine, et que le tracé de la carte géologique résulte peut-être d'une extrapolation.
On ne peut s'empêcher d'évoquer le rejeu d'une très ancienne faille qui, plus à l'ouest, s'infléchit pour devenir parallèle au Connecticut, et qui, plus au sud encore, forme un des côtés du fossé triasique qui traverse l'État du même nom.

Cependant, on ne saurait préciser son rejet, faute de disposer comme repère d'une surface d'aplanissement bien identifiée. Cette dislocation doit remonter à un âge assez reculé et avoir été exagérée par l'érosion différentielle. Dans le compartiment supposé déprimé, l'intrusion syénitique de Cherry Mountain dépasse $1000 \mathrm{~m}$.

Entre le coude du Connecticut et le massif des White Mountains, le relief se compose de croupes grossièrement parallèles NE-SW, alternant avec des dépressions. Si l'on excepte quelques dorsales quartzitiques, elles sont en position oblique par rapport aux contacts lithologiques. Tout se passe comme si l'érosion glaciaire avait exploité des bandes plus fracturées parallèles aux failles et chevauchements majeurs remontant à l'orogenèse acadienne.

Dans toute cette partie septentrionale, qui culmine entre 1000 et $1500 \mathrm{~m}$, le relief paraît peu dépendant de la nature des roches: différentes variétés de syénites et de granites des White Mountains Series, et aussi, plus à l'ouest, les monzonites quartzifères dites de Kinsman, mises en place au Dévonien, et dont la position haute n'est pas justifiée par leur composition minéralogique (plagioclases relativement abondants).

Les accidents topographiques les plus remarquables sont de profondes rainures de direction méridienne. Elles correspondent à des lignes de fracture recoupant des variétés intrusives de toutes catégories, parmi lesquelles figurent des roches très résistantes comme des granites alcalins à hastingsite. Franconia Notch recoupe des granites de Conway ayant entraîné des enclaves de gneiss olivériens. Dans le fond de l'auge, on peut vérifier que certains affleurements sont fortement fissurés, soit à l'échelle de la diaclase, soit à l'échelle microscopique, d'où leur perméabilité au soluchrome, tandis que sur le flanc ouest, les mêmes roches donnent un dôme monolithique. Crawford Notch tranche les migmatites d'injection lit par lit. L'étranglement maximal de la vallée de la rivière Saco est formé par une syénite à grain fin, réduite à un mince ruban à peu près parallèle à la rainure.

La raideur des versants semble due au fait que l'exploitation des lignes de faiblesse a été un phénomène tardif (intervention des glaciers et des eaux fluvioglaciaires). Dans la partie méridionale du même massif, les alvéoles s'élargissent et la différenciation du relief paraît en rapport avec la variété pétrographique des intrusions mézosoïques.

Dans sa partie méridionale, le massif des White Mountains (s.s.) s'abaisse progressivement. De plus, il est aéré par des dépressions d'abord fermées, puis ouvertes vers la vaste région déprimée qui s'étend jusqu'à 
l'Océan. Elles paraissent avoir été excavées par l'érosion différentielle.

Les syénites et les granites hypersolvus occupent toujours une position culminante, à la fois en raison de leur composition minéralogique et de leur faible perméabilité.

Au SE de la cuvette de Bartlett axée sur des fractures $\mathrm{EW}$, un paquet de rhyolites qui, postérieurement à leur mise en place, ont subi un réchauffement et une recristallisation de la pâte, est traversé par des syénites porphyroïdes qui donnent l'escarpement des Attitash Mountains. La cloison occidentale est constituée de syénites ou d'un granite hypersolvus renfermant plus de $70 \%$ de perthites où l'albite colmate toutes les irrégularités. Quelques fissures dans le quartz sont comblées par des amorphes. Au SW, Mount Tremont est formé d'un granite à riébeckite où la trame des feldspaths alcalins riches en soude forme l'essentiel de la roche. Des lattes de plagioclases séparent les microclines, mais sont rarement en contact avec les cristaux de quartz groupés en nids. Les dalles de base du massif sont également très résistantes; la roche y est difficile à classer, renfermant à la fois des feldspaths potassiques, de l'augite et de l'olivine, ainsi que de la biotite (plutôt du clan des syénites).

A la base de certains versants, on observe parfois des affleurements de granite rose en mauvais état, du faciès Conway.

La cuvette de Passaconaway est traversée par des entailles évasées entre lesquelles affleurent des roches résistantes. A l'ouest, les Loon Mountains sont formées d'un granite à riébeckite et hastingsite, avec $50 \%$ de feldspaths alcalins: par ailleurs, sa perméabilité au soluchrome est nulle, même sur les affleurements superficiels. La perméabilité à l'eau (procédé Berneix) est inférieure à $10^{-13}$. Le granite de Conway n'affleure qu'à la base du versant ouest; il est très diaclasé et en état d'altération déjà avancée. Si la carte géologique attribue ces cuvettes au granite de Conway, c'est probablement à partir de la composition de la moraine, supposée autochtone, et aussi en fonction des observations déjà citées, faites à la base des versants.

En fait, les surfaces attribuées à cette unité pétrographique révèlent à l'examen des faciès très variés. D'une part, des granites roses où dominent les perthites (55 à 60\%) et, d'autre part, des granites gris, riches en plagioclases et en biotite (et qui sont peut-être des enclaves très larges de granite dévonien?).II ne faut donc pas s'étonner si le comportement de ces roches vis-àvis de l'érosion différentielle présente d'assez grandes variations, comme l'indiquent les faits relevés dans la région de Conway elle-même.

Tout le secteur de Conway (s.s.) est caractérisé par l'orientation méridienne des reliefs, suivant de grandes lignes de fracture exploitées par l'érosion glaciaire. La plus remarquable est le corridor de Conway où les contrastes lithologiques jouent un rôle relatif moins important. Au nord, il suit en effet des syénites qui sont fissurées, sauf au passage du banc qui détermine la chute de Jackson. Au moment de rejoindre le corridor, un banc massif de granite de Conway rose provoque une autre chute (Glen). II se trouve en contact assez brutal avec un granite gris, tant par la couleur de ses feldspaths que par l'abondance des éléments ferromagnésiens. Ce dernier type de roche est complètement arénisé. Le granite rose lui-même présente certaines bandes arénisées, du type de celles qui soutiennent le "monument» naturel, dit Peyro Clabado (Sidobre, SW du Massif Central français); elles ne peuvent s'expliquer que par la fissuration.

Plus au sud, un escarpement légèrement arqué, de $400 \mathrm{~m}$, correspond au contact avec les laves de Moat inclinées vers l'intrusion du granite de Conway. Ce dernier présente localement un faciès gris. L'intrusion suit une fracture, qui a rejoué postérieurement à sa mise en place (coupe 4, fig. 1).

Le versant oriental de l'auge complexe présente des pentes plus faibles. Le massif qui est traversé par Harrison Trail montre des alternances de granite gris et de granite rose assez fortement diaclasés.

A ces reliefs modestes s'opposent des monolithes de granite rose coupés par des fractures méridiennes (Cathedral Ledge, Redstone). Ils sont découpés par des diaclases courbes formant des lentilles biconvexes ou biconcaves. Mais la microfissuration n'est pas anormalement faible: comme en témoigne une microporosité de $1 \%$ au porosimètre à mercure, et une perméabilité à l'eau Berneix de 10-9. II existe également des monolithes de granites riches en éléments ferromagnésiens au SE de South Conway.

Au total, ces monolithes semblent avoir été individualisés ou tout au moins exagérés par leur résistance à la poussée du bulldozer glaciaire, qui entraînait les volumes plus fortement diaclasés. La face orientale concave de Cathedral Ledge - demi-dôme recoupé par une fracture méridienne - correspond probablement à la matrice, au moule laissé par une lentille rocheuse transportée par les glaciers en direction du sud.

Grâce à un sondage effectué au voisinage de Redstone (WARREN et TIERNAU, 1981), I'hétérogénéité du granite de Conway apparaît dans les trois dimensions (fig. 8). Sur les 300 premiers mètres, se rencontre le faciès gris; sur les $400 \mathrm{~m}$ suivants, le faciès rose, pauvre en éléments ferro-magnésiens. Enfin, jusqu'à la base située à $1000 \mathrm{~m}$, se retrouve le faciès gris vert (tabl. VI).

On peut se demander s'il est légitime de considérer comme appartenant à la même unité des volumes rocheux aussi disparates et au contact si nettement tranché, en l'absence d'arguments chimiques ou radiochronologiques. 


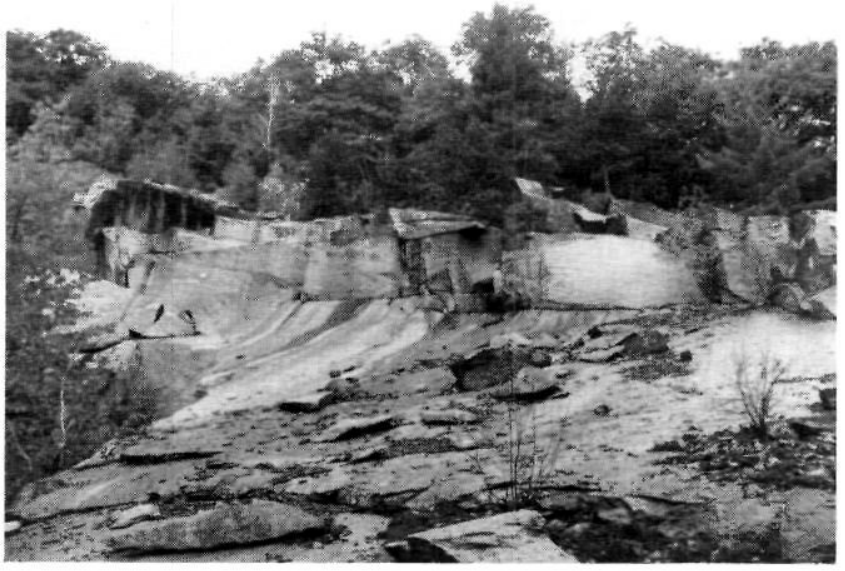

FIGURE 8. Sur le flanc SW de Redstone Ledge, le granite très massif de Conway: ancienne carrière de Redstone à $4 \mathrm{~km}$ SSE de North Conway.

On the SW side of Redstone Ledge, the very massive Conway granite; former Redstone quarry, $4 \mathrm{~km}$ SSE of North Conway.

\section{TABLEAU VI}

Composition minéralogique des granites de Conway (en pourcentage) d'après le sondage de Redstone

\begin{tabular}{|c|c|c|c|c|c|}
\hline $\begin{array}{l}\text { Profondeur } \\
\qquad(\mathrm{m})\end{array}$ & Q & FK & $\mathrm{PI}$ & $\mathrm{Bi}$ & $A m$ \\
\hline \multirow{4}{*}{200} & \multicolumn{5}{|c|}{ Faciès de Conway (s.s.) } \\
\hline & \multicolumn{5}{|c|}{ Faciès gris-vert (dit Osceola) } \\
\hline & \multicolumn{5}{|c|}{ 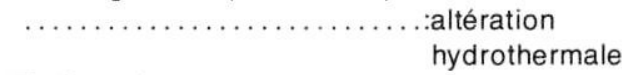 } \\
\hline & \multicolumn{5}{|c|}{ Faciès gris-vert } \\
\hline 300 & 32 & 38 & 24 & 4 & \\
\hline 335 & 10 & 13 & 43 & 10 & 13 \\
\hline & \multicolumn{5}{|c|}{ Faciès rose de Conway (s.s.) } \\
\hline 380 & 37 & 51 & 6 & & \\
\hline 448 & 27 & 56 & 13 & & \\
\hline 528 & 35 & 46 & 12 & \\
\hline 700 & 16 & 50 & 22 & 7 & \\
\hline 770 & \multicolumn{5}{|c|}{ Faciès gris-vert au-dessous de $770 \mathrm{~m}$} \\
\hline
\end{tabular}

Quoi qu'il en soit, ce sondage a aussi l'intérêt de montrer l'existence de volumes altérés hydrothermalement, où la roche est tendre et perméable, par exemple, entre 117 et $190 \mathrm{~m}$.

Le sondage de Redstone a été exploité dans le but de définir la microfissuration des différents volumes rocheux prospectés tant par l'étude de la propagation des ondes que par l'examen microscopique. Malheureusement, les résultats du traitement statistique ont une valeur affaiblie par le petit nombre des échantillons considérés (8 à 10).

Cependant, on remarque deux groupes de roches. Les unes sont faiblement microfissurées, car la vitesse des ondes de compression augmente peu avec la pression hydrostatique de confinement (profondeur: 300 ,
448, 523), tandis que le résultat est inverse pour le groupe II $(704,381,335)$. On trouve des granites fissurés dans la variété rose comme dans la variété grise. Cette hétérogénéité du granite de Conway rend compte du fait à prime abord déconcertant que de vastes cuvettes aèrent surtout la partie centrale des White Mountains.

Plus à l'est encore, le complexe intrusif se termine par un grand escarpement méridien long de $25 \mathrm{~km}$, qui le sépare des collines de piedmont du massif du Mount Washington. II recoupe une gamme de roches très variées (granite de Conway, granite à hastingsite, syénite, etc.). Dans sa moitié méridionale, l'érosion différentielle suffit à rendre compte du relief, puisque la dépression correspond au granite dévonien. Au nord de Catham, la carte géologique indique des séries métamorphiques dérivant de carbonates, qui, assez souvent, résistent moins bien que les autres horizons des séries de Littleton; un doute reste quant à leur extension véritable, car elles ne sont pas individualisées sur la carte géologique voisine du Maine (à l'échelle de $1 / 500000$, il est vrai).

Quoi qu'il en soit, le caractère rectiligne de l'escarpement suggère l'intervention de failles recoupant le complexe intrusif, mais peut-être assez anciennes. Dans le dernier cas, l'escarpement serait inversé par érosion différentielle (?)

À l'ouest du corridor complexe de Conway, le rebord méridional du massif acquiert une grande vigueur (6 à $700 \mathrm{~m}$ de dénivellation). Sans doute est-il renforcé par de larges intrusions de granite à hastingsite et hedenbergite. Le piedmont est constitué tantôt de granite de Conway, tantôt de séries pélitiques de Littleton, dont ni la composition ni le degré de métamorphisme n'indiquent de différence sensible avec celles qui culminent au Mount Washington. Même s'il s'agit de schistes "ordinaires", l'escarpement excède en vigueur celui que l'érosion différentielle justifierait: est-il permis d'évoquer l'effet direct d'une faille avec redans?

Plus à l'ouest encore, on a affaire à la lente retombée du massif des White Mountains se comportant comme un bloc basculé vers le sud, et nivellant tour à tour les granites de Conway, les monzonites de Kinsman et les séries de Littleton.

Si l'hypothèse d'un escarpement de faille était retenue pour le secteur de Wonalancet, il faudrait supposer que ce compartiment occidental s'abaisse en touche de piano vers l'est.

Quoi qu'il en soit, le piedmont méridional est formé de collines dont la hiérarchie a été interrompue par des coups de gouge glaciaires. Constituées tantôt par les séries de Littleton, elles cèdent la place à des alvéoles en grande partie occupés par des lacs, coïncidant presque toujours avec de vastes intrusions de diorites quartzifères plus ou moins foliées et d'âge supposé dévonien; très localement, elles s'enrichissent en quartz et en feldspaths potassiques. 
L'érosion différentielle n'a pas dit son dernier mot. Elle a mis clairement en relief la structure annulaire d'Ossipee Mountain (600 m de dénivellation), dont l'armature est formée par les laves des séries de Moat, traversées par les intrusions hypovolcaniques plus récentes; les éléments les plus résistants sont des roches d'affinité rhyolitique, dont les deux phases sont composées entièrement de quartz et de feldspaths alcalins. Le mince ruban arqué de granites porphyriques à amphiboles participe également à la construction du relief. Ici encore, les granites de Conway, les plus tardifs, occupent une position ambiguë dans l'échelle de dureté, car s'ils coïncident avec le cœur déprimé du complexe, ils participent également à la partie interne de la couronne en saillie.

\section{DISCUSSION DES PRINCIPALES CONCLUSIONS}

\section{A. L'ÉCHELLE DE DURETÉ}

1. Les corps en saillie

Ils correspondent principalement aux intrusions et, accessoirement, aux laves d’âge mésozoïque.

a) La composition minéralogique (tabl. III, IV et V).

Ce facteur est assurément important. II s'agit de roches pauvres en minéraux altérables.

La position culminante des rhyolites, dont l'effusion marque le début de la mise en place des structures annulaires, ne saurait surprendre. Tout concourt à ce résultat: la rareté des plagioclases (s.s.) et de la biotite - le grand développement des perthites dans les phénocristaux - l'existence d'une pâte microgrenue, très imperméable, recristallisée lors des intrusions.

La fissuration des phénocristaux (probablement d'origine thermique) est scellée par des dépôts d'oxyde de fer.

Parmi les roches plutoniques, les syénites occupent une position dominante. II n'y a pas d'exemple de syénite en creux (sauf à l'intérieur de l'intrusion de Brome). D'autre part, parmi les granites "acides" où prédominent les minéraux résistant à l'altération chimique (quartz et feldspaths potassiques et sodiques), pour une même valeur de la somme de ces deux éléments, ce sont les intrusions récentes mises en place à haute température, et où l'albite est associée à l'orthose dans les perthites, qui sont les plus dures. Tout se passe comme si l'albite des perthites ou microperthites n'était pas une cause de faiblesse, à la différence de celle qui est engagée dans les plagioclases s.s. (dont la composition, dans le cas qui nous intéresse, est celle de l'oligoclase). Un exemple est celui du Mount Tremont, à l'ouest de Bartlett, riche en albite. La même relation apparaît dans le comportement vis-à-vis de l'érosion différentielle de la petite intrusion circulaire post-dévonienne de Tunk Lake qui fait partie du groupe de la côte du Maine. KARNER (1968) a signalé l'expression orographique de l'anneau granitique externe qui domine de $200 \mathrm{~m}$ une dépression intérieure accidentée toutefois par une bosse centrale, sauf là où la roche a été hachée de fractures dans l'arc méridional. II résulte de nombreuses analyses modales que le granite externe est plus pauvre en quartz, mais beaucoup plus riche en perthite que le granite interne. Quant aux minéraux ferro-magnésiens, il s'agit d'ægyrine ou de hornblende dans le premier cas, et de biotite dans le second. Mais comme leur pourcentage ne dépasse pas 3, leur influence sur l'altération est négligeable. La composition chimique montre que la teneur en calcium du centre est presque aussi faible que celle de la périphérie. En définitive, la faiblesse des granites centraux se ramène au fait que $\mathrm{Na}$ est présent dans les plagioclases, et non pas dans les perthites (KARNER et BERTRAM, 1972).

Par conséquent, l'échelle de dureté à l'intérieur des White Mountains Series n'est pas conforme à l'échelle de solubilité des minéraux, telle qu'elle devrait intervenir si on multipliait la teneur de chacun d'entre eux par sa vitesse de solubilisation, une unité de quartz comptant pour 10 unités de feldspath potassique, ellemême égale à 5 ou 6 unités d'albite (BIROT, 1981).

On est conduit à imaginer qu'il existe des liens de valeur inégale entre les contacts des différents cristaux, l'albite étant très fortement liée à l'orthose au sein des perthites, les relations les plus lâches étant celles qui unissent le quartz et le plagioclase. Dans l'état actuel de nos connaissances, il est impossible de justifier cette situation. Nous ne savons presque rien sur les énergies de surface au contact de deux cristaux.

\section{b) La perméabilité}

Ce cortège de roches est en même temps peu perméable, spécialement les syénites et les granites à amphiboles ou pyroxènes, tandis que les granites de Conway ont un comportement plus irrégulier. Ces faits peuvent être confrontés avec les conditions de mise en place des intrusions.

Rappelons que la perméabilité des corps plutoniques est liée à une micro-fissuration qui, elle-même, peut être l'héritage d'une porosité «aqueuse" acquise à la fin de la cristallisation du magma, selon l'hypothèse proposée par l'un d'entre nous (BIROT, 1972, 1981; BIROT et GUITARD, 1971). On imagine que Iorsqu'un magma riche en eau cristallise, une partie de la vapeur supercritique libérée n'a pas la possibilité de s'échapper par un toit trop vite consolidé, et qu'elle reste sur place, déterminant des vides qui compromettent la solidité de la roche.

Malheureusement, l'évaluation de cette teneur en eau à partir des données minéralogiques et chimiques est très difficile. On dispose sans doute d'un éventail d'expériences pétrogénétiques dont la multiplication permet d'espérer qu'un jour on pourra disposer d'un nombre suffisant d'équations pour déterminer simultanément la 
pression, la température et la teneur en eau dans la chambre de cristallisation. La composition des feldspaths n'est guère influencée par ce dernier facteur pour des pressions de l'ordre de $2 \mathrm{~kb}$. II en va autrement pour de très fortes pressions; alors le rapport Ab/Or est d'autant plus petit que le magma est plus sec. L'ordre de cristallisation des granites ne dépend pas de la teneur en eau pour les pressions relativement basses $(2 \mathrm{~kb})$ : se déposent successivement les plagioclases, les feldspaths alcalins et les quartz. Mais pour les fortes pressions, cet ordre est changé dans les magmas très secs où l'on a PI-Q-Fa et dans les magmas très humides: Fa-Q-PI.

Même lorsque ces équations seront établies, il faudra être sûr que l'ordre des cristallisations n'est pas une manifestation d'un métasomatisme postmagmatique, ce que l'examen microscopique ne suffit pas toujours à déterminer.

En attendant, on peut admettre que l'absence de minéraux hydroxylés, ou la présence de minéraux faiblement hydroxylés comme les amphiboles, lorsqu'ils cristallisent tardivement, sont des signes de sécheresse du magma. On constate que les granites à biotite des intrusions mésozoïques sont moins résistants que les granites à amphiboles et pyroxènes, toutes choses égales d'ailleurs quant au pourcentage modal de ces minéraux ferro-magnésiens (qui demeure, en général, trop faible pour intervenir directement dans la sensibilité à la solubilisation). La présence de minéraux pauvres en hydroxyles, lorsqu'ils cristallisent tardivement, est le seul signe utilisable du caractère relativement sec des magmas parentaux (voir aussi BAILLY, 1968). Certains des granites de Conway, qui ont cristallisé en dernier lieu, se sont relativement enrichis en eau, ce qui leur a permis d'acquérir une certaine porosité.

Cependant, pour que les pores primaires se transforment en fissures, il est nécessaire que la région subisse de fortes contraintes de compression. Or, les intrusions considérées sont naturellement postérieures à l'orogenèse majeure. Pour que le mécanisme joue, il faudrait admettre que les mouvements verticaux postjurassiques se sont accompagnés d'une importante compression.

D'autre part, les intrusions les plus anciennes ont pu subir, au moins dans des volumes réduits, une fissuration d'origine thermique. En effet, Iorsqu'un corps plutonique envahit un corps plus ancien imperméable, son refroidissement se fait surtout par conduction. D'après les modèles de NORTON et KNIGHT (1977), un cylindre de $5 \mathrm{~km}$ de rayon réchauffe au bout de 100000 ans un anneau périphérique de $3 \mathrm{~km}$ de large jusqu'à des températures comprises entre 200 et $400^{\circ} \mathrm{C}$. Le cyclindre soumis aux mêmes isothermes au-dessus des intrusions mesure environ $2 \mathrm{~km}$ de hauteur (NORTON et KNIGHT, 1977).

Or, suivant les expériences de PERAMI (1971), on peut estimer que la fracturation se produit principale- ment entre 200 et $300^{\circ} \mathrm{C}$. Pour des températures plus élevées, des cristaux soumis à des contraintes commencent à réagir par déformation plastique.

Ces contraintes peuvent avoir une double origine: a) l'inégalité des coefficients de dilatation des cristaux, suivant les espèces minérales et, pour une même espèce minérale, suivant l'orientation des plans des cristaux. C'est ce mécanisme qui a fait l'objet des expériences de PERAMI (1971) et des travaux de WILHEM (1977). b) La cristallisation du corps magmatique qui se refroidit provoque des phénomènes de tension sur les roches encaissantes, puisque la somme du volume des cristaux et du volume de l'eau supercritique libérée est supérieure à celui du magma.

La fissuration a pour conséquence de permettre l'organisation d'une circulation d'eau convective, avec réchauffement suivant le trajet $A B$, et refroidissement ensuite entre $B$ et $C$. Le long du trajet $A B$, la capacité de mise en solution des quartz et des feldspaths va augmentant. $A 7 \mathrm{~km}$ de profondeur et $400^{\circ} \mathrm{C}$, la solubilité des feldspaths alcalins est environ de $2 \mathrm{~g} / \mathrm{l}$, et celle du quartz de $1,5 \mathrm{~g} / \mathrm{l}$. Or, à $200^{\circ} \mathrm{C}$, elle se réduit à 100-150 mg/l. Par conséquent, au cours du trajet CD, les fissures auront tendance à se boucher (fig. 9).

Mais ici, il faut prendre en considération l'eau qui s'échappe du magma et qui avait été libérée par la cristallisation. Pour un magma humide, elle peut représenter $5 \%$ en poids. Elle est naturellement en équilibre vis-à-vis des cristaux, et se trouve par conséquent très

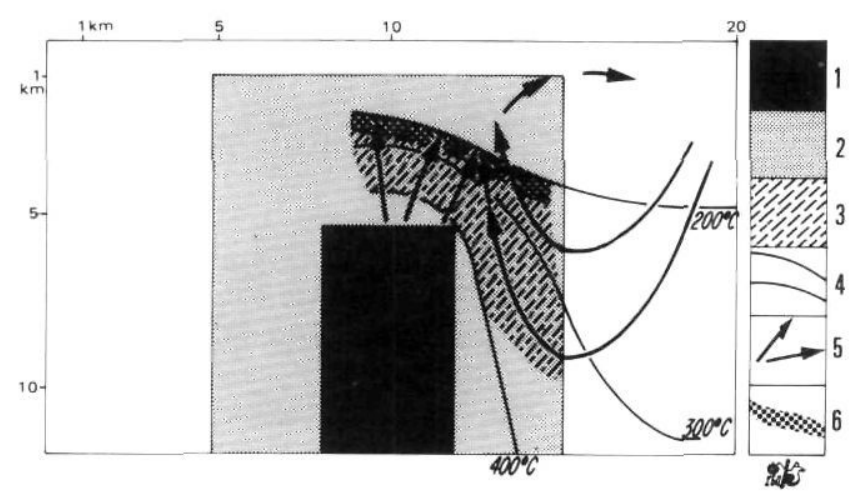

FIGURE 9. Schéma des possibilités de fissuration et de circulation de l'eau dans des intrusions emboîtées. 1) Nouvelle intrusion - 2) Ancienne intrusion - 3) Volumes fissurés - 4) Isothermes 1 million d'années après l'intrusion, en supposant que le refroidissement s'est fait par conduction - 5) Circulation de l'eau dans le volume fissuré - 6) Couronne où les dépôts de produits cristallisés l'emportent sur les départs en solution.

Model of genesis of the fissuration and the water-flow lines into intrusive stacked bodies. 1) New intrusive - 2) Older intrusive - 3) Thermally-fissured bodies - 4) Isotherms 1 million years after the intrusion (cooling controled by conduction) -5) Waterflow-lines through the fissured body -6) Top crown where recrystallisation exceed solution. 
chargée en $\mathrm{Si}, \mathrm{K}$ et $\mathrm{Na}$. Ce flux constituera le principal agent d'obturation des fissures situées au-dessus de la partie supérieure du corps plutonique récent. Ce départ d'eau crée des vides à l'intérieur de l'intrusion récente elle-même. Tel est le seul mécanisme qui paraît capable de justifier l'augmentation de perméabilité dont fait état un des modèles de Norton (NORTON et KNAPP, 1977).

Dans nos intrusions annulaires, ces mécanismes n'ont joué qu'un rôle mineur, en fissurant, sur quelques centaines de mètres de large, les syénites les plus anciennes.

\section{Les corps plutoniques en creux (tabl. I)}

Les corps plutoniques en creux, dont le grand axe va de quelques kilomètres à quelques dizaines de kilomètres, sont considérés comme d'âge dévonien ou plus ancien.

Leur composition minéralogique dominante est celle des granodiorites ou des diorites quartziques. Cependant, sur des surfaces plus ou moins réduites, ils sont enrichis en feldspaths potassiques cicatrisant des déformations ou des fissures, soit par métasomatisme, les fluides ascendants provenant d'une intrusion plus profonde, soit par suite de la montée jusqu'à la surface d'un magma neuf plus siliceux que le précédent. De tels volumes sont mis en saillie (Long Mountain). En moyenne, ils sont plus perméables que les corps plutoniques récents. En effet, ils ont pu acquérir dans de meilleures conditions une porosité "aqueuse" parce que, lorsqu'ils se sont mis en place par fusion aux dépens d'un encaissant schisteux, ils ont pu s'enrichir en ions $\mathrm{OH}$. D'autre part, ils ont subi au moins la fin des compressions de l'orogenèse acadienne: d'où des possibilités de fissuration.

Dans la mesure où se sont produites, en particulier au cours du Dévonien, plusieurs bouffées magmatiques, les nouvelles intrusions sont susceptibles d'avoir provoqué une fissuration thermique. Nous avons vu que celle-ci a été, au moins partiellement, scellée par le dépôt entraîné par des fluides ascendants ayant leur origine dans des intrusions sous-jacentes à la surface actuelle du sol. Enfin, ces petits batholites sont en général entourés d'une auréole de métamorphisme thermique dont les cornéennes sont très résistantes.

À plusieurs reprises, il est apparu que les cuvettes et alvéoles calqués sur les intrusions dévoniennes exploitaient des roches minéralogiquement fragiles même massives, tandis que les bourrelets et échines de bordure se calaient sur des roches de l'encaissant parfois fort divisées (cuvette du lac aux Îles, cuvette de Nulhegan River etc.). Tout se passe comme si l'évidement était à mettre au compte, pour l'essentiel, d'un système morphogénique à météorisation chimique prédominante. Si d'indiscutables perturbations ont été introduites localement, par le passage des glaciers, elles n'ont pas modifié fondamentalement la distribution des reliefs et des creux acquise antérieurement. Cette conclusion n'est pas sans rappeler l'interprétation que suggère l'examen morphologique des corps intrusifs écossais (GODARD, 1965).

Cependant, des intrusions de même âge, affleurant sur une surface dont le diamètre dépasse à peine $1 \mathrm{~km}$, sont au contraire plus dures que les schistes encaissants. II s'agit du toit de granites à deux micas, dont la bordure figée est souvent identifiable.

Le travail d'exhumation ne fait que commencer à Barre, près de Montpellier (Vermont). Le granite à deux micas, d'âge sans doute dévonien, est troué par une carrière géante (fig. 10). L'horizon supérieur est enveloppé par des diaclases à très grand rayon de courbure séparées de plusieurs mètres et recoupées par des diaclases verticales également très espacées. Si les noyaux des blocs intermédiaires sont parfaitement imperméables (perméabilité Berneix $=10^{-13}$ ), les volumes plus externes absorbent assez facilement le saluchrome. Le chapelet des dômes du mont SaintSébastien est beaucoup mieux dégagé des schistes. II possède les mêmes propriétés quant à la perméabilité de diaclase et à la microperméabilité. Un facteur supplémentaire de dureté est le développement des perthites.

La démonstration que seul le banc granitique du toit est résistant se confirme lorsqu'on examine une série d'intrusions dévoniennes de granite à deux micas, semblable à celui de Barre, et située à la frontière du Québec et du Vermont (Beebe-Graniteville). Grâce à de nombreuses carrières, on possède une coupe où une carapace résistante d'une dizaine de mètres surmonte les granites du cœur intrusif qui est déprimé, tant par rapport au banc sommital que par rapport à l'auréole métamorphique.

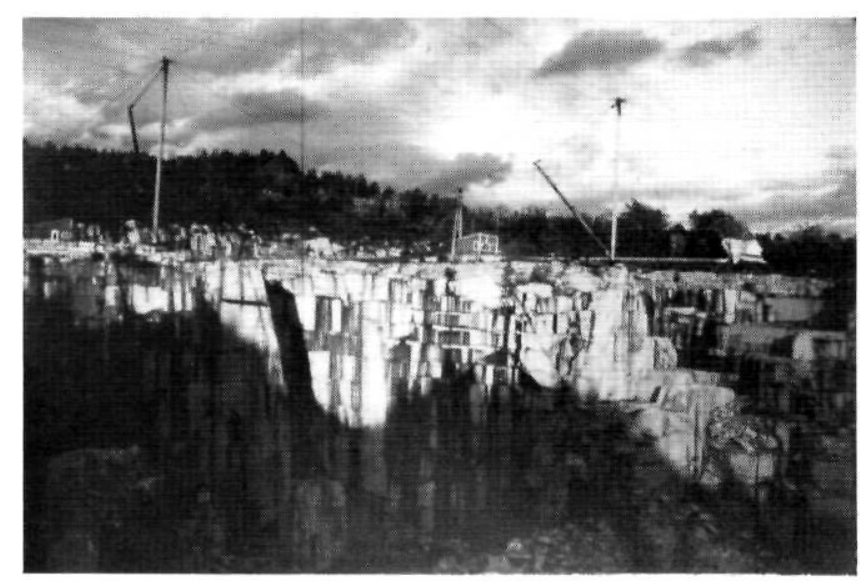

FIGURE 10. Le dispositif orthogonal des diaclases dans les profondes carrières de granite de Barre (Vermont).

The orthogonal joint system in the deep granite quarries in Barre (Vermont). 


\section{Les roches feuilletées}

Les roches feuilletées du domaine considéré se répartissent dans toute l'échelle de dureté, depuis les argilites et les calcaires non métamorphiques de la région de Montréal, les formations olivériennes et Ammonoosuc, jusqu'aux séries de Littleton. Si l'on excepte les cas extrêmes de roches se rapprochant des calcaires marneux, ou au contraire des quartzites, il existe une large gamme où la composition minéralogique ne constitue pas le facteur décisif: par exemple, la plupart des gneiss olivériens sont moins riches en minéraux altérables que les gneiss de base des séries de Littleton.

Apparemment, tout dépend d'une aptitude inégale au découpage en plaquettes, c'est-à-dire de la force des liens qui unissent les microlithons à travers les plans de schistosité ou de foliation. Si ces liens sont faibles dès l'époque du métamorphisme, ou affaiblis par des contraintes de compression postérieures à la cristallisation qui provoque le décollement des microlithons, d'autant plus facilement que les plans de schistosité sont exactement parallèles, la roche se comporte comme relativement tendre. $\mathrm{Si}$, au contraire, la cristallisation continue au-delà de la période où s'exercent les contraintes tectoniques, il est à supposer que, d'une part, les liens chimiques entre cristaux parallèles sont améliorés, et que, d'autre part, des cristaux transverses à la schistosité diminuent le caractère anisotrope de la structure, donc augmentent la résistance à la compression.

Ceci posé, on peut rechercher la logique des dispositions structurales d'ensemble qui permettent de prévoir la répartition des roches feuilletées dans une grande unité structurale dont l'axe central subit un réchauffement:

1) Les horizons inférieurs de la série sédimentaire soumise à un métamorphisme dynamo-thermique restent exposés pendant un temps relativement plus long à de hautes températures (par exemple 300 à $500^{\circ} \mathrm{C}$ ), qui permettent le «recuit»; ainsi disparaissent les trains de dislocations qui avaient fragmenté les grains lors des premiers stades du métamorphisme, tandis qu'est réalisé un meilleur équilibre entre les cristaux superposés de nature différente.

Au contraire, toutes choses égales d'ailleurs quant à la durée de la compression tectonique, les horizons supérieurs ne sont touchés que pendant moins longtemps par l'onde de hausse des températures. Ces dernières restent, au total, plus faibles, et les microfractures ne sont qu'incomplètement scellées par la recristallisation. D'après les travaux de VOISIN (1981), il est possible d'expliquer ainsi la faible résistance des phyllites de la Famenne (Ardenne belge).

2) Si nous considérons ce qui se passe dans les horizons inférieurs, la recristallisation est plus ou moins complète suivant le bilan des importations et des expor- tations de matières dissoutes de la circulation hydrothermale (d'après des principes analogues à ceux qui ont été proposés dans le cas d'une intrusion magmatique); la différence est que, dans le sens horizontal surtout, l'échelle des distances est plus petite lorsque le réchauffement affecte une large colonne sédimentaire et que ses effets s'atténuent progressivement de part et d'autre.

Là aussi doivent s'organiser des circuits convectifs. L'eau superficielle descend, puis se rapproche de l'air surchauffé, pour remonter ensuite. Pendant la première partie du trajet, la dissolution chimique crée des vides, alors que dans la seconde partie, les vides antérieurs sont bouchés. Au-dessus de l'air réchauffé, il faut ajouter des colonnes ascendantes de vapeur correspondant à la déshydratation de la muscovite, à partir de $500-550^{\circ} \mathrm{C}$. Elles s'attaquent aux minéraux dans la zone de départ, et tendent à combler les vides dans les horizons où les isothermes se tiennent vers $200-300^{\circ} \mathrm{C}$.

$\mathrm{Ce}$ modèle permet de prévoir que les roches feuilletées sont inégalement compactes, sans qu'il soit d'ailleurs question de vérifier sa validité dans un cas concret. Même en les soumettant à des tests statistiques, il ne suffit pas de procéder à quelques analyses chimiques comparées d'une série sédimentaire normale et de son équivalent soumise à un métamorphisme de haut degré, pour établir un bilan convaincant du résultat, comme il a été tenté pour les séries de Littleton (tabl. V).

Nous avons pu vérifier par l'exemple des séries de Littleton que les phénomènes de rétro-métamorphisme ne constituent pas nécessairement une cause d'affaiblissement. L'intervention d'une deuxième phase de fracturation entraînant la reprise de la circulation d'eau ne rend pas le délitage plus facile, lorsque le bilan des apports et des exportations de produits dissous est positif et lorsque la température est assez élevée pour permettre une formation suffisamment rapide des nouveaux cristaux.

\section{B. RÓLE RESPECTIF DES SOULĖVEMENTS VERTI- CAUX, DES APLANISSEMENTS ET DE L'ÉROSION DIFFÉRENTIELLE}

Les aplanissements atteignent leur maximum de perfection dans la région de Montréal, en fonction du niveau de base du Saint-Laurent, mais il est vrai dans des roches peu résistantes - et d'autre part, dans les collines à très larges alvéoles du piedmont atlantique, où elles ne sont surmontées que par quelques chicots ou barres de roches dures: quartzites de Boote et granites des intrusions de Tunk et du Mount Desert.

Dans l'espace intermédiaire s'étendent des reliefs à première vue anarchiques, en réalité surtout contrôlés par l'érosion différentielle (sauf quelques gorges et surcreusements glaciaires) et surtout le massif des White Mountains (s.l.) qui, certes, se compose de roches placées au sommet de l'échelle de dureté, mais dont les 
contours d'ensemble suggèrent l'héritage d'un bloc faillé du côté NW, et basculé vers l'Atlantique, peut-être vers le milieu du Tertiaire. Si cette faille se place bien à la limite SE des formations olivériennes, l'escarpement est à la fois d'origine tectonique et dû à l'exploitation d'un contact de dureté.

Les photographies aériennes, montrant la pyramide du Mount Washington comme posée sur un socle de croupes situées vers 1000 à 1200 m, pourraient suggérer que celles-ci dérivent d'une surface d'aplanissement ayant presque complètement arasé les roches les plus dures, surface inclinée à la fois vers le SE et le SW. En réalité, elles ne portent aucun aplanissement vraiment net. Et on peut douter que le temps de stabilité nécessaire à son élaboration ait été imparti à la région, si on essaie de calculer la tranche de roche qui a été enlevée depuis la mise en place des intrusions mésozoïques.

En l'absence de toute couverture enregistrant les soulèvements, reste la possibilité d'évaluer l'épaisseur de la tranche érodée depuis la mise en place des corps intrusifs.

Ici, nous rencontrons une première méthode: celle qui prend en compte les associations de minéraux de métamorphisme en équilibre, caractéristiques d'une certaine profondeur (dans l'hypothèse où les contraintes tectoniques sont relativement négligeables par rapport à la pression hydrostatique). La carte des bathogrades ainsi dressée (CARMICHAEL, 1978) pour les séries pélitiques métamorphosées au Dévonien, dans le nord de la Nouvelle-Angleterre implique l'ablation d'une tranche de $17 \mathrm{~km}$ par l'érosion. Telle est, en effet, la pression critique correspondant à l'équilibre: andalousite $=$ kyanite + sillimanite .

Il faudrait en conclure que cette région n'a jamais connu de phase de stabilité suffisante pour permettre le développement d'une surface d'aplanissement généralisée (sans cependant de dénivellations suffisantes pour engendrer un contraste bioclimatique).

La tranche érodée diminue rapidement d'épaisseur en direction du nord-est. Dans l'est du Maine et le Nouveau-Brunswick, l'association quartz-muscovitesillimanite-feldspath potassique-andalousite indiquerait une profondeur inférieure à $8 \mathrm{~km}$. De plus, le corps granitique de Red Beach, à la frontière du NouveauBrunswick, se serait mis en place à 1,5 km seulement, à en juger par la teneur en oxydes de fer, les biotites et feldspaths alcalins.

Quelles que soient les séductions de cette méthode, il faut cependant rester prudent quant à sa fiabilité. En effet, le même bathograde serait d'âge pensylvanien ou permien à Rhode Island, ce qui laisse peu de temps pour l'érosion d'une tranche de roches aussi importante, puisque nous savons que cette région a été aplanie dès le Trias.

Des conclusions plus précises et plus intéressantes sont fournies par des minéraux datés radiométrique- ment, et pour lesquels on calcule l'épaisseur probable des terrains surincombants. Nous disposons à cet égard d'une série de données récentes.

Les intrusions des White Mountains Series ont fait l'objet de trois types de datations: 1) Les datations $\mathrm{K} / \mathrm{Ar}$ indiquent l'époque à laquelle la biotite s'est "fermée ". La température, à ce moment, était de $300-200^{\circ} \mathrm{C}$. 2) L'examen des traces de fission des zircons (avec une température critique de $200^{\circ} \mathrm{C}$ ). 3) Les traces de fission de l'apatite, avec une température critique de $100^{\circ} \mathrm{C}$.

Par ailleurs, on a calculé d'après les modèles déjà anciens la vitesse de refroidissement d'un dôme plutonique. Si l'on attribue à ce dernier une température initiale de $700^{\circ} \mathrm{C}$ à $750^{\circ} \mathrm{C}$, le refroidissement s'arrête au bout de 1 à 5 millions d'années seulement, au cœur d'un cône de $1,5 \mathrm{~km}$ de rayon. Cela signifie qu'après 10 millions d'années la température de la plus grande partie de l'intrusion a retrouvé la température initiale de l'ensemble des terrains encaissants. Cela exprime à peu près la différence d'âge entre les données K/Ar et celles qui correspondent au commencement de l'accumulation des traces de fission dans le zircon. On en déduit que la température des terrains encaissants était de $200^{\circ} \mathrm{C}$ pour les intrusions jurassiques datées de 180 M.A. (Conway Granite, et aussi la syénite du Mount Monadnock). En admettant un gradient thermique de $25-30^{\circ} \mathrm{C} / \mathrm{km}$, cela donne une distance de la surface du sol de $7 \mathrm{~km}$. Si l'apatite a un "âge" de 115 millions d'années, c'est-à-dire crétacé, c'est que le soulèvement et l'érosion consécutive ont, dans l'intervalle, réduit à $3 \mathrm{~km}$, l'épaisseur des terrains superposés (DOHERTY et LYONS, 1980).

Cela signifie, qu'à la différence des massifs hercyniens européens, le nord des Appalaches aurait été soumis à des soulèvements et à une érosion intense, tant au cours du Mésozoïque qu'à l'ère tertiaire, où un régime de bourrelet marginal montagneux a du exister. L'épaisseur de la tranche érodée diminuait d'ailleurs sensiblement en direction du bourrelet canadien.

On s'explique dès lors le faible développement des surfaces d'aplanissement, très médiocrement représentées sur le versant atlantique. Pendant les quelques dizaines de millions d'années disponibles, l'érosion n'a pu remplir sa tâche que si les versants des vallées sont restés presque constamment jeunes.

\section{SUMMARY}

Despite the inconvenient fact that the bedrock is partly hidden beneath a glacial drift, this area was chosen for the variety of the lithological types.

Our goal was to ascertain the relative influence of mineralogical composition versus permeability. The previous modal analysis have been collected and completed by our own sampling. Permeability, related to microfracturation has been appraised by microscopic 
examination, and measurement of the rate of filtration of coloured water.

Between Montreal and Granby, a true inselberg-plain, locally covered by pleistocene sediments, cuts across calcareous shales and schists, which of course are weaker than Monteregian cretaceous plugs, ranging from syenites to more mafic essexites, gabbros and yamaskites. But inside the inselbergs, some hollows have no geological control.

In the Eastern Townships and NE Vermont, the schists are truncated by a peneplain. Granitic Devonian intrusives are in many instances hollowed out into basins, which can be explained either by their granodioritic composition (Island Pond), or by their microfissuration (Scotstown).

The prominent position of the White Mountains (s.s.) appears as chiefly (but not exclusively) due to selective weathering, sparing a complex of harder rocks units, fortuitously juxtaposed: the White Mountains Jurassic intrusive and Littleton Series. Their NW boundary coincides with the oliverian gneisses which are easily eroded because of incomplete recrystallisation after fracturation. So are the Bickford granites (despite their rather acid composition) intruded into Littleton Series. However the hardness of the latter (if understood where quartzites outcrop) is uneasily explained where we are dealing with gneisses and, a fortiori, metapelites. The prominent position of the rhyolites, syenites and hastingsite or riebeckite granites is a well-known fact in Corsica, Africa, etc. The other types of granites (classified as Conway, Osceola, etc.) are heterogeneous in their mineralogical composition ranging from acid massively jointed to gray microfractured varieties with abundant plagioclases, biotites and hornblende; it may explain the topographic contrast between huge monoliths and basins (where unfortunately the outcrops are scarce).

The SE boundary of the White Mountains coincides with the appearance of the Maine peneplain, where, however, the duration of the tectonic rest was too short for allowing the Mesozoic intrusives to be planated. This hypothesis is supported by a recent evaluation of the Mesozoic and Tertiary erosion rates.

\section{Discussion}

Most of the differential erosion was accomplished before the inlandsis invasion, which could do not more than excavate some closely jointed rock volumes. The features previously described are due to chemical weathering, mainly acting during the Tertiary. The top of the scale of hardness to weathering is occupied by rocks of which perthites and microperthites are the main components, juxtaposed in a continuous web: i.e syenites and hypersolvus granites. Moreover, it is suggested that chemical bonds are stronger between albite and microcline or orthose than between albite and anorthite - and relatively weak between quartz and plagioclases.
The roofs of the granitic bodies are impervious and not fissurated, which may be related to the hypothesis that recrystallisation is supposed to be more active than solution by the ascending fluids escaping from magma: so, previous microfractures are sealed. On the contrary solution outpaces recrystallisation in the cores of several granitic bodies.

Cauldron-like low areas, excavated in Devonian granodioritic bodies are easily explained by the abundance of calcic plagioclases, biotite or hornblende.

The behavior of foliated rocks depends more upon their permeability than their mineralogical composition. The bonds of the tiny plates are strong if crystallisation keeps up later than tectonic compression (for instance in the large zones of contact with intrusives in NE Vermont) and weak in the reverse case.

\section{REMERCIEMENTS}

Les auteurs remercient le Centre national de la recherche scientifique (C.N.R.S., France), le Conseil des arts (Canada) et les universités de Sherbrooke et de Montréal pour l'aide matérielle qui leur a été accordée dans la préparation et le déroulement des campagnes d'étude sur le terrain. Madame $M$. Ters a prêté son concours pour l'examen pétrographique des lames minces. $\mathrm{Cl}$. Bernard, P. Clément et D. Pilon ont participé à plusieurs déplacements sur le terrain. II convient enfin d'associer à ces remerciements les collaborateurs (techniciens et administratifs) du L.A. 141 et le dessinateur du L.A. 260.

\section{BIBLIOGRAPHIE}

BAILLY, B. (1968): Introduction to Petrology, Prentice Hall, New Jersey.

BILLINGS, M.P. (1937) : Regional metamorphism of the LittletonMoosilauke area, New Hampshire, Geol. Soc. Am. Bull., vol. 48, p. $463-566$.

(1941): Structure and metamorphism in the Mount Washington area, New Hampshire, Geol. Soc. Am. Bulll., vol. 52, p. 863-936.

BILLINGS, M.P. et WILLIAMS, C.R. (1938): Petrology and structure of the Franconia quadrangle, New Hampshire, Geol. Soc. Am. Bull., vol. 49, p. 1011-1043.

BIROT, P. (1972): Recherche d'un modèle rendant compte de l'inégale perméabilité des roches cristallines, Recherches géogr. en France, Montréal, 1972, p. 47-52.

BIROT, P. (1981): Les processus d'érosion à la surface des continents, Paris, $600 \mathrm{p}$.

BIROT, P. et GUITARD, G. (1971) : Observations sur le relief du socle hercynien des Pyrénées orientales, Rev. Géogr. des Pyrénées et du S.O., t. 42, fasc. 1, p. 5-30.

BIROT, P., GODARD, A., PETIT, M. et TERS, M. (1974) : Contribution à l'étude des surfaces d'aplanissement et de l'érosion différentielle dans le Transvaal septentrional et oriental (Afrique du Sud), Rev. Géogr. phys. Géol. dynamique (2), vol. XVI, fasc. 4, p. 421-454. 
BLANCHARD, R. (1960): Le Canada français, Lib. A. Fayard, Paris-Montréal, 316 p., 35 fig., 12 p. h.t.

CARMICHAEL, D.M. (1978): Metamorphic bathozones and bathograds: a measure of the depth of post-metamorphic uplift and erosion on the regional scale, Am. Journ. of. Science, vol. $278, n^{\circ} 6$, p. 769-797.

CHAPMAN, R.V. (1935): Intrusions in Percy Quadrangle, Am. Journ. of Science, vol. 30, p. 401-431.

(1948): Geology of the Percy quadrangle, Am. Journ. of Science, vol. 59, p. 1059-1100.

CHAPMAN, C.A. et CHAPMAN, R.V. (1944): Petrology and structure of the Oliverian magma series in the Mount Washington quadrangle, New Hampshire, Geol. Soc. Am. Bull., vol. 55, p. 497-516.

CHAPMAN, C.A., CHAPMAN, R.V., FOWLER-BILLINGS, K. et LOOMIS, F. B. Jr. (1946): Geology of the Mount Washington quadrangle, New Hampshire, Geol. Soc. Am. Bull., vol. 57, p. 261-273.

CHAPMAN, C.A. et RABBIT, J.C. (1947) : Chemical analyses and calculated modes of the Oliverian Magma series, Mount Washington quadrangle, New Hampshire (Shaler Memorial Series), Geol. Soc. Am. Bull., vol. 58, p. 573-596.

CLÉMENT, P. et DE KIMPE (1977): Geomorphological conditions of gabbro weathering at Mount Megantic, Quebec, Can. Journ. Earth Sci. 14, p. 2262-2273.

CLÉMENT, P. et PELLETIER, J. (1980): Le mont Mégantic (Québec) : essai de mise au point géomorphologique, Géogr. phys. Quatern. vol. 34, p. 175-185.

CZAMANSKE, G.K., WONES, D.R. et EICHELBERGER, J.C. (1977): Mineralogy and petrology of the intrusive complex of the Pliny Range, New Hampshire, Am. Journ. Science, vol. 277 , p. 1073-1123.

DIMENT, W.H. (1968): Gravity anomalies in Northwestern New England, Studies of Appalachian Geology Northern and Maritime, p. 399-414.

DOHERTY, J.T. et LYONS, J.B. (1980): Mesozoic erosion rates in Northern New England, Geol. Soc. Am. Bull., part I, vol. 91, $\mathrm{n}^{\circ}$ 1, p. 16-20.

DOUGAN, T.W. (1979): Compositional and modal relationships and melting reactions in some migmatitic metapelites from Hampshire and Maine, Am. Journ. of Science, vol. 279, p. 897-935.

GODARD, A. (1965): Recherches de géomorphologie en Écosse du Nord-Ouest, Strasbourg, $701 \mathrm{p}$.

KARNER, F.R. (1968): Compositional variation in the Tunk Lake Granite Pluton, Southeastern Maine, Geol. Soc. Am. Bull., vol. 79, n 2, p. 193-222.

KARNER, F.R. et BERTRAM, R.E. (1972): Modal variation in granitic units of the White Mountains plutonic-volcanic series, New Hampshire, Intern. Geol. Cong. Canada, Section 2, Pédologie, Montréal, p. 164-170.

MARMÖ, V. (1971): Granite petrology and the granite problem, Developments in Petrology, 2, Amsterdam-Londres-New York, Elsevier Publ. Co., 244 p.
NAYLOR, R.S. (1968): Origin and regional relationships of the core-rocks of the Oliverian domes, Studies Appalachian Geology Northern and Maritime, p. 231-240.

(1969): Age and origin of the Oliverian domes, Central Western New Hampshire, Geol. Soc. Am. Bull., vol. 80, n ${ }^{\circ}$, p. $405-428$.

NORTON, D. et KNAPP, R. (1977): Transport phenomena in hydrothermal systems: the nature of porosity, Am. Journ. of Science, vol. 277, $n^{\circ} 8$, p. 913-936.

NORTON, D. et KNIGHT, J. (1977): Transport phenomena in hydrothermal systems: cooling plutons, Am. Journ. of Sciences, vol. $277, n^{\circ} 8$, p. 937-981.

PAGE, L.R. (1968): Devonian plutonic rocks in New England, Studies of Appalachian Geology Northern and Maritime, p. 371-384.

PERAMI, R. (1971): Recherches sur la microfissuration thermique des roches, Thèse Univ. de Toulouse.

PERRAULT, G., édit. (1970): Alkaline rocks: the Monteregian Hills, $598 \mathrm{p}$.

QUINN, A. (1944): Magmatic contrasts in the Winnipesaukee region, New Hampshire, Bull. Geol. Soc. Am., vol. 55, p. 473496.

REID, A. M. (1976) : Rapport sur la géologie du mont Mégantic, Min. Richesses naturelles du Québec.

RITCHOT, G. (1975): Essais de géomorphologie structurale, Presses Univ. Laval, Québec, 588 p.

SHAW, D.M. (1956): Geochemistry of pelitic rocks, Part III: Major elements and general geochemistry, Bull. Geol. Soc. Am. vol. 67, $\mathrm{n}^{\circ}$ 7, p. 919-934.

THOMPSON, J.B., Jr. et NORTON, S.A. (1968): Paleozoic Regional Metamorphism in New England and adjacent areas, Studies of Appalachian Geology Northern and Maritime, p. 319-328.

THOMPSON, J.B., Jr., ROBINSON, P., CLIFFORD, T.N. et TRASK, N.J., Jr. (1968): Nappes and gneiss domes in WestCentral New England, Studies of Appalachian Geolog Northern and Maritime, p. 203-218.

VOISIN, L. (1981): Le modelé schisteux en zone froide et tempérée, Thèse Univ. Paris IV, $498 \mathrm{p}$.

WARREN, N. et TIERNAU, M. (1981): Systematics of Crock controlled mechanical properties for a suite of Conway granites, Tectonophysics, t. 73, p. 295-333.

WILHEM, S. (1977): Étude minéralogique des feldspaths en relation avec leur contexte géologique (héritage magmatique et rôle des phénomènes thermiques dans la formation des microtextures) Thèse, Univ. Paris VI.

WILHEM, S. et WILLAINE, C. (1976): A propos des fentes dites d'extension des phénocristaux felspathiques des granites et des gneiss. Rôle des contraintes thermiques, C.R. Ac.Sc. Paris, série D, vol. 282, n 3 , p. 255-258.

ZEN, E. A., WHITE, W. S., HADLEY, J. B. et THOMPSON, J. B., Jr., édit. (1968), Studies of Appalachian geology Northern and Maritime, Interscience New York, 451 p. 